\title{
Esofagitis necrotizante (esófago negro). Presentación de casos y revisión de la literatura
}

\section{Necrotizing esophagitis (black esophagus): Presentation of cases and literature review}

Luis Alberto Ángel Arango, MD, ${ }^{1}$ Exided Jesús Cortés Mora, MD, ${ }^{2}$ Elías Alfonso Forero Piñeros, MD, ${ }^{3}$ Héctor José Cardona Villamizar, MD³

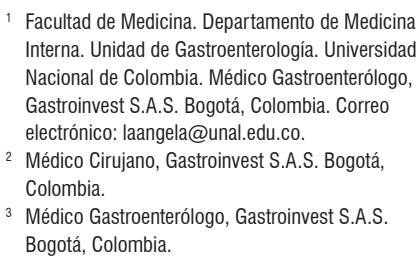

1 Facultad de Medicina. Departamento de Medicina Interna. Unidad de Gastroenterología. Universidad Nacional de Colombia. Médico Gastroenterólogo, Gastroinvest S.A.S. Bogotá, Colombia. Correo electrónico: laangela@unal.edu.co.

2 Médico Cirujano, Gastroinvest S.A.S. Bogotá Colombia.

${ }^{3}$ Médico Gastroenterólogo, Gastroinvest S.A.S. Bogotá, Colombia.

Fecha recibido: $13-10-17$ Fecha aceptado: 26-07-18

\begin{abstract}
Resumen
La esofagitis necrotizante aguda (esófago negro) es una patología grave poco frecuente, que se caracteriza por presentar pérdida parcial o total del epitelio, con ulceración hasta el esfacelo circunferencial de todas las capas de la mucosa y submucosa. También se evidencia compromiso frecuente de las capas profundas musculares y perforación, con aspecto negruzco al examen endoscópico, que le ha dado su nombre más conocido, el de esófago negro. Se presentan cinco casos ilustrativos concordantes con la descripción de la literatura médica, que luego de ser revisada reportó todos los casos descritos, junto con los factores de riesgo y pronóstico.
\end{abstract}

\section{Palabras clave}

Esofagitis necrotizante, esófago negro, necrosis esofágica aguda, descripción de casos, revisión, factores de riesgo.

\section{Abstract}

Acute necrotizing esophagitis (black esophagus) is a rare pathology that is characterized by partial or total loss of the epithelium, ulceration up to the circumferential slough of all layers of the mucosa and submucosa, frequent involvement of deep muscle layers, and frequent perforations. Its blackish appearance in endoscopic examinations has given it the name of black esophagus. Five illustrative cases, all concordant with descriptions the medical literature, are presented in this study together with a review of the literature, case descriptions and risk and prognosis factors.

\section{Key words}

Necrotizing esophagitis, black esophagus, acute esophageal necrosis, case description, review, risk factors.

\section{INTRODUCCIÓN}

La esofagitis necrotizante aguda es una patología poco frecuente, que cursa con alteración de gravedad variable en la mucosa, desde la pérdida parcial del epitelio con ulceración hasta el esfacelo de todas las capas de la mucosa y compromiso de las capas profundas musculares y perforación. También presenta necrosis focales o extensas y que, por la coloración negruzca al examen endoscópico, le han dado su otro nombre, el más conocido, de esófago negro. Existe poca literatura acerca del tema, se desconoce su fisiopatología y fácilmente puede confundirse con quemaduras por cáusticos, precisamente por su aspecto, solo que sin dicho antecedente. Los dos primeros casos fueron descritos por Brennan (1) y Lee y colaboradores (2) en pacientes con necrosis esofágica extensa y en paciente con hipotermia severa y ruptura aórtica espontánea con infarto esofágico extenso, respectivamente.

Dado su carácter dramático, desde el punto de vista endoscópico, y su cuadro clínico y posible desenlace, se decidió realizar una revisión de la literatura médica disponible. 


\section{SUJETOS Y MÉTODOS}

Se describen cinco casos de pacientes con diagnóstico endoscópico de esofagitis necrotizante aguda o esófago negro, atendidos en centros hospitalarios de la ciudad de Bogotá, con su evolución clínica (si estaba disponible), los cuales fueron comparados con los hallazgos proporcionados por la literatura.

La revisión se realizó mediante buscadores electrónicos en las bases de datos PubMed, Scielo, Google, Ovid, Hinari, Springer, Ebsco y Sinab (Universidad Nacional), mediante los términos "esófago negro", "black esophagus", "black oesophagus", "necrotizing" AND "oesophagitis", "necrotizing” AND "esophagitis", "esofagitis necrotizante" y en la bibliografía referida en los artículos encontrados. Todos los artículos fueron aceptados si estaban en inglés, francés, español y portugués, o por lo menos el resumen (abstract) en alguno de estos idiomas. Se encontró una revisión sistemática de la literatura realizada por Day y Sayegh, de 2010 (3), que incluyó todos los casos descritos hasta 2008, y se procedió a describir los casos informados desde entonces hasta agosto de 2017, para luego consolidarlos con la tabla de los autores mencionados y en el mismo formato, para hacerlos comparables y describir adecuadamente la totalidad de los casos.

\section{DESCRIPCIÓN DE LOS CASOS}

\section{Caso 1 (JL)}

Mujer de 79 años, que consulta por urgencias por melanemesis y alteración de la conciencia. Como antecedente pre-

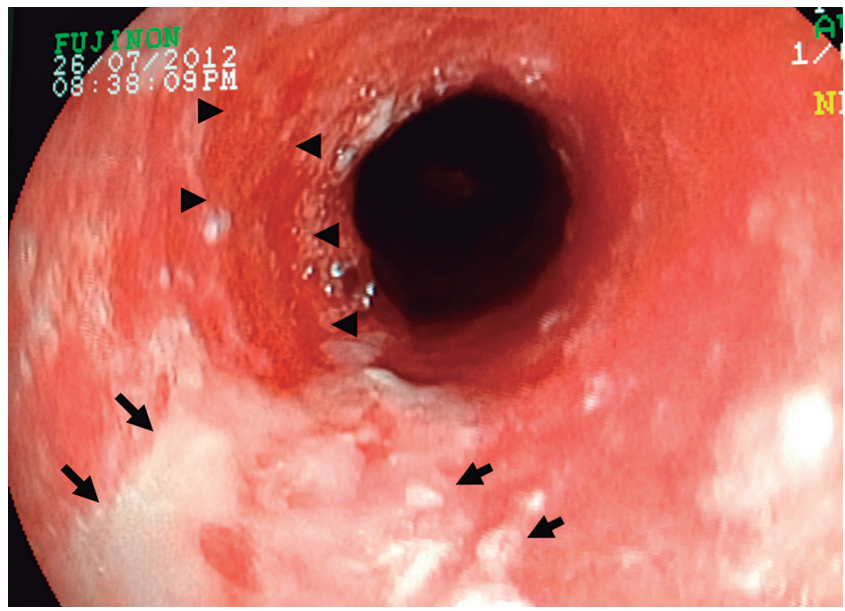

Figura 1. Fragmentos de mucosa esfacelada en el tercio medio proximal del esófago (flechas) y en las zonas extensas descubiertas cruentas (cabeza de flechas) (caso 1). sentaba meningioma resecado, con síndrome convulsivo secundario, hipertensión arterial no controlada y enfermedad pulmonar obstructiva crónica. En los exámenes presentaba leucocitosis con neutrofilia (89\%); creatinina de $1,84 \mathrm{mg} / \mathrm{dL}$, hipocloremia $(92 \mathrm{mEq} / \mathrm{L}$ ) e hiponatremia $(129 \mathrm{mEq} / \mathrm{L})$. La endoscopia mostró esfacelo del epitelio esofágico en los dos tercios inferiores y sangrado difuso en la capa (Figuras 1, 2, 3 y 4), sin cambios en el calibre. Se desconoce su evolución y su desenlace.

\section{Caso 2 (MDA)}

Mujer de 79 años, que asiste a control por antecedente de hemorragia digestiva y que es atendida por urgencias con hallazgos endoscópicos de moniliasis proximal y con ulceración circunferencial de aspecto hematínico rojo, pardo y negro, con áreas de necrosis, con friabilidad y sangrado fácil al contacto con el endoscopio en el tercio medio y distal, con mucosa severamente edematosa y rígida, sin estenosis (no hay documento fotográfico). Las alteraciones desaparecen a partir de la línea $\mathrm{Z}$, la cual se halla ascendida a los $32 \mathrm{~cm}$; hiato esofágico amplio con hernia hiatal gigante, con diagnóstico de esofagitis severa sugestiva de lesión por cáusticos. Las biopsias practicadas en el esófago distal muestran una esofagitis aguda con extensa necrosis mucosa y submucosa, con capa muscular viable; cambios epiteliales reactivos. La paciente y los familiares negaron la posibilidad de ingestión de cáusticos, con antecedentes de anemia ferropénica cuatro años atrás y endoscopias previas por hemorragia digestiva, con hernia hiatal de $9 \mathrm{~cm}$ y erosiones de Cameron uno y cuatro años anteriores al episodio actual. El control endoscópico un mes después muestra

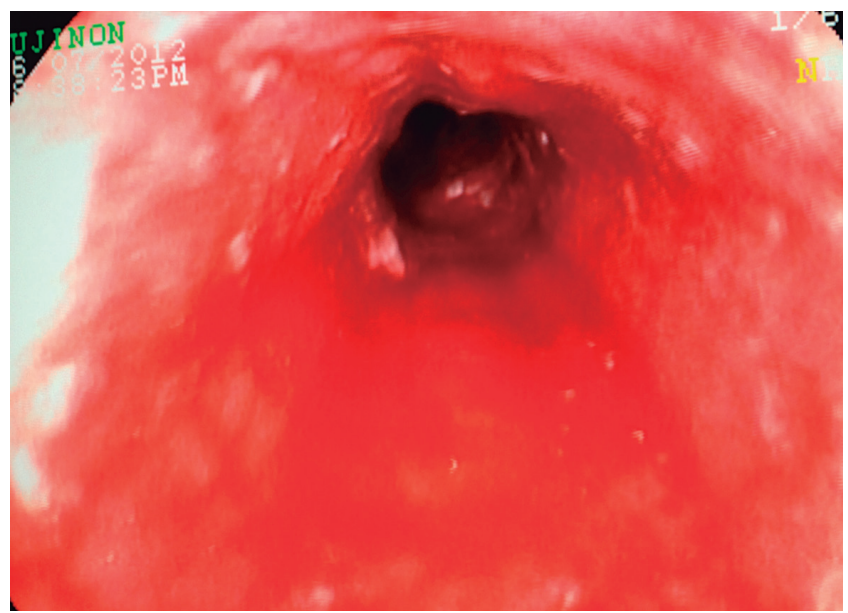

Figura 2. Esfacelo difuso de la mucosa del tercio medio del esófago, con sangrado en capa (caso 1). 


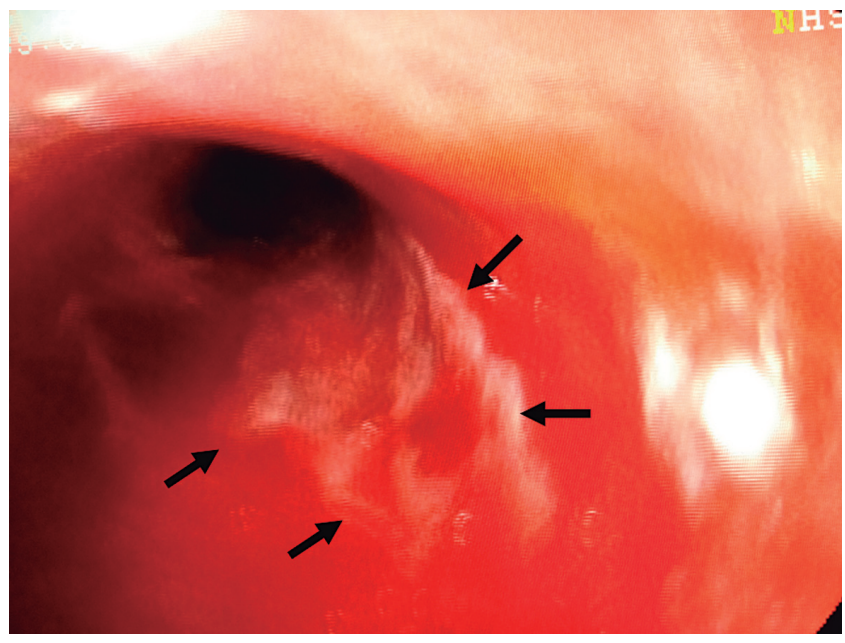

Figura 3. Fragmentos de mucosa esofágica esfacelada en el tercio medio (flechas) y sangrado en capa (caso 1).

una hernia hiatal gigante de $9 \mathrm{~cm}$ y gastritis erosiva crónica antral, con gastritis crónica no atrófica inactiva leve en las biopsias antrales.

\section{Caso 3 (HS)}

Varón de 76 años, que fue remitido por consultar por dolor intenso en el hipocondrio derecho, de tres días de evolución, malestar general, astenia y adinamia. $\mathrm{Al}$ examen estaba adolorido, taquicárdico, taquipneico, afebril, ictérico, con tirajes intercostales, estertores en base derecha y signo de Murphy positivo. Antecedente de diabetes mellitus tipo 2 e hipertensión arterial. Con diagnósticos de neumo-

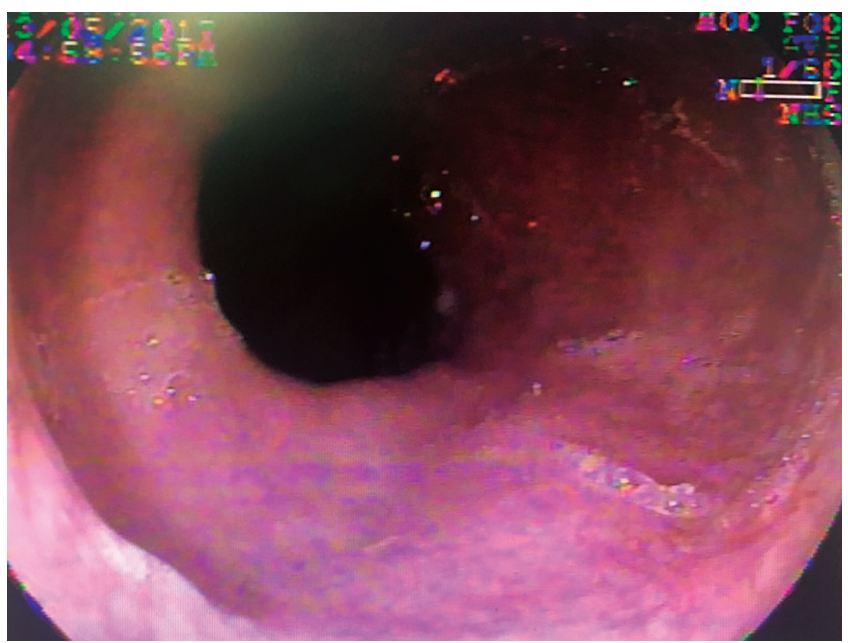

Figura 5. Esófago cervical normal (caso 3).

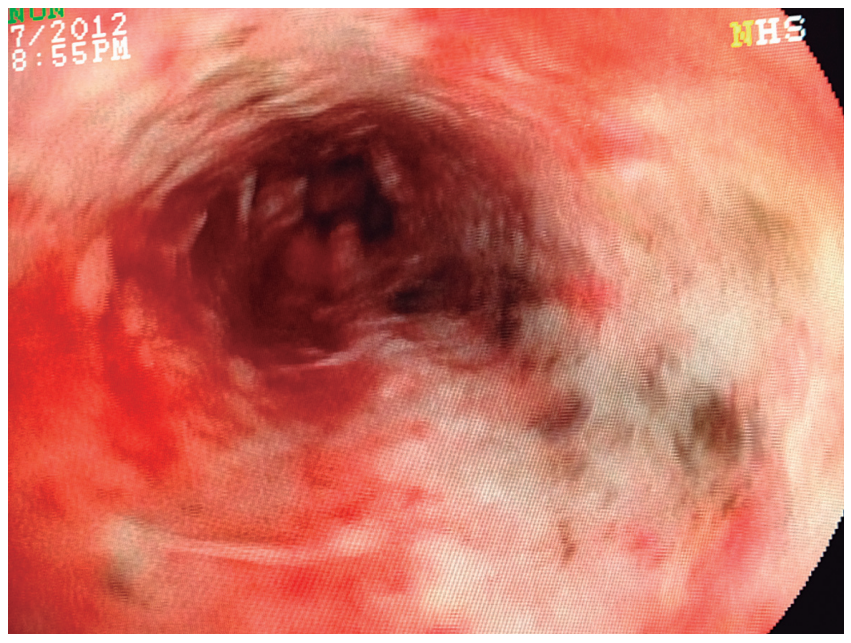

Figura 4. Esfacelo difuso de la mucosa esofágica con ulceración extensa, focos de necrosis en el tercio distal y zonas de sangrado en capa (caso 1).

nía basal derecha, septicemia, trombocitopenia, hipertrofia prostática benigna, insuficiencia renal crónica agudizada, colelitiasis con dilatación de la vía biliar y colédocolitiasis. Durante la ultrasonografía endoscópica, como hallazgo incidental se describe mucosa esofágica negra en su totalidad, recubierta de exudado mucoso, sin erosiones, úlceras ni estrechez, con mucosa por debajo de la línea $\mathrm{Z}$ de aspecto usual. La videoendoscopia demuestra ulceración esofágica desde los $21 \mathrm{~cm}$ de la arcada dentaria, que se torna circunferencial a los $23 \mathrm{~cm}$, recubierta por fibrina, aspecto negro y grisáceo desde los $27 \mathrm{~cm}$ hasta la unión cardioesofágica (Figuras 5, 6 y 7), respetando la mucosa cardial (Figura 8). La colangiopancreatografía retrógrada endoscópica

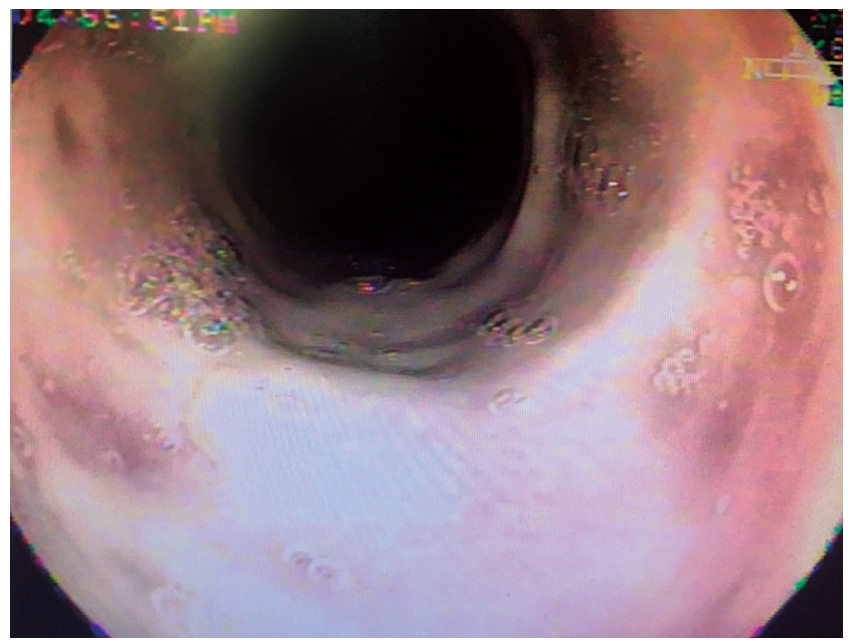

Figura 6. Ulceración esofágica proximal circunferencial recubierta por fibrina (caso 3). 


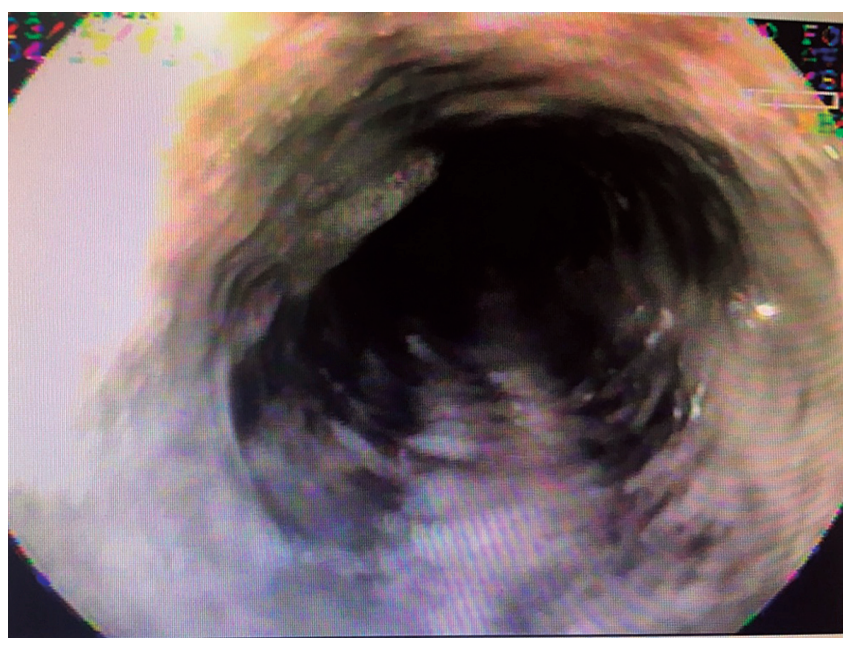

Figura 7. Esófago negro y grisáceo distal (caso 3).

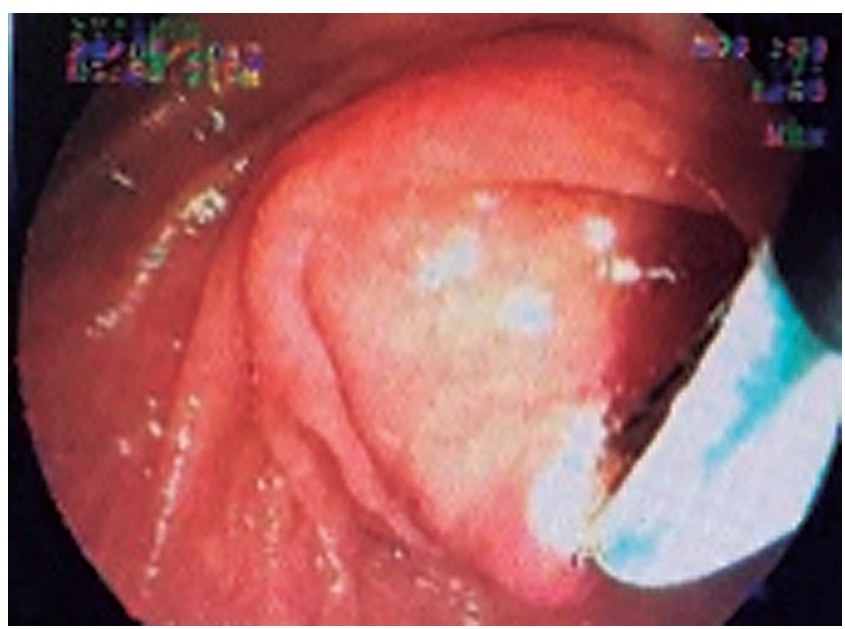

Figura 9. Esfinterotomía pequeña para drenaje para extracción de cálculos (caso 3).

(CPRE) demuestra vía biliar extrahepática dilatada con defecto de llenado en el tercio medio y distal; se practica esfinterotomía pequeña (por trombocitopenia de $31000 \mathrm{x}$ $\mathrm{mm}^{3}$ ) (Figura 9) y se extraen cálculos pequeños con balón, dejando un stent plástico de $7 \operatorname{Fr} \times 7 \mathrm{~cm}$, con drenaje de bilis clara con microcálculos. El paciente evoluciona satisfactoriamente y sale del hospital con resolución del cuadro doloroso e ictérico. A las 10 semanas se encuentra asintomático, sin aceptar la realización de procedimientos de control.

\section{Caso 4 (JMR)}

Varón de 81 años, con diagnóstico de trombosis mesentérica y hemorragia digestiva, en quien se solicita endoscopia

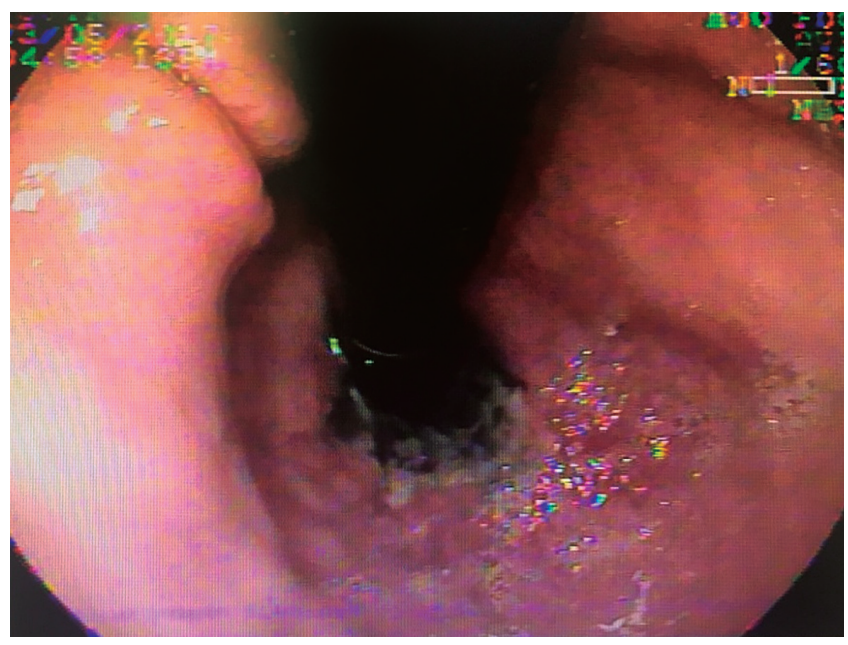

Figura 8. Unión cardioesofágica en retrovisión, con cardias respetado (caso 3).

digestiva que muestra mucosa esofágica en toda su extensión con zonas de necrosis, hemorragia, lesiones ulceradas recubiertas por fibrina, sin sangrado activo, ni signos de perforación y con línea Z irregular ubicada a los $38 \mathrm{~cm}$ de la arcada dentaria. Con diagnósticos de necrosis panesofágica y pangastritis crónica. No se cuenta con registro fotográfico ni evolución del paciente.

\section{Caso 5 (MECL)}

Mujer de 73 años, con diagnóstico de hemorragia digestiva, en quien se solicita endoscopia digestiva que muestra gran hematoma en la pared posterior del esófago, con disección de la mucosa y sangrado espontáneo. Hay mucosa en el tercio medio, con zonas de necrosis y caída de fragmentos mucosos, con tercio distal normal. No se cuenta con registro fotográfico ni evolución de la paciente.

\section{DISCUSIÓN}

\section{Epidemiología}

La esofagitis necrotizante o esófago negro es una condición rara, descrita inicialmente en necropsias de casos aislados $(1,2)$ y en series de autopsias, con una frecuencia del $0 \%$ al 0,02\% $(4,5)$, y más recientemente 32 casos de necrosis esofágica en una serie de 310 autopsias, para una incidencia del 10,3\% (6). Ulteriormente, con la aparición de la endoscopia, según los autores su frecuencia ha variado entre el $0,01 \%$ y el $0,28 \%$ de las endoscopias (7). Grudell reportó una incidencia del 0,008 \% (6 casos en 78847 endoscopias) (8); Ramos la describió en el 0,28 \% de 3976 endoscopias consecutivas (9); Gómez la informó en el 0,11\% de 
6011 endoscopias consecutivas (10); mientras que Yasuda reportó este hallazgo en el $6 \%$ de las endoscopias realizadas por hemorragia digestiva aguda en un período de tres años (11). Nuestros casos no permiten establecer la frecuencia relativa, dado que son casos aislados en instituciones de tercer nivel, aunque en una casuística de 12638 endoscopias realizadas en un centro de diagnóstico de segundo nivel no se encontró ningún episodio (12), lo que refleja la gravedad de la entidad, tal como se describe en la literatura de comorbilidad asociada en el $83 \%$ de los casos $(3,7)$. Hay predominio del género masculino y de la edad avanzada, particularmente en la séptima y octava décadas $(3,7)$, con 78 años promedio en nuestros pacientes y tres mujeres (60\%), para 70 años y dominancia masculina (3:1) en la serie total (Tabla 1).

\section{Presentación clínica}

Los casos descritos en la literatura ascienden a 261 (266 con los nuestros), con descripción aislada de casos en la inmensa mayoría y algunas series grandes de 29 (13) y 16 casos (11). Solo se encontraron tres revisiones extensas de la literatura. En la primera, Grudell y colaboradores, en 2006 (8), recopilaron los primeros 52 casos de esófago negro entre 1963 y 2003; en la segunda, Gurvitts y colaboradores, en 2007 (81), recopilaron 88 casos adicionales; y en la tercera, realizada por Day y Sayegh, los autores recopilaron los casos restantes hasta el 2008, que sumaron a los anteriores (3). En las Tablas 1 y 2 se observan los datos y hallazgos de todos los casos descritos en la literatura $(3,8-11,13-81)$.

Como puede verse, nuestros pacientes corresponden a adultos mayores, todos hospitalizados, cuya indicación principal fue la hemorragia digestiva alta $(4 / 5)$, corroborándose el sangrado activo en tres, al igual que los casos totales (Tabla 1) y otros menos frecuentes, como disfagia, choque, anemia, inconciencia y muerte súbita. En nuestra serie, el diagnóstico fue incidental en un paciente durante el estudio de patología de vía biliar complicada.

El diagnóstico es esencialmente endoscópico, ocasionalmente por cirugía de urgencia o autopsia $(1,2,4)$ (Tabla 1). La gravedad de la lesión presentó compromiso circunferencial en cuatro y con zonas de necrosis en cuatro pacientes; la localización fue difusa en los casos 3 y 4, dos tercios distales en los casos 1 y 2 , y tercio medio en el caso 5 , acorde con la serie general, en la que el $73 \%$ presentaba compromiso entre los dos tercios y la totalidad del esófago (Tabla 1). Sin embargo, su apariencia es similar a la esofagitis cáustica, que fue sospechada en un caso y descartada clínicamente por el paciente y los familiares, demostrándose ulteriormente que ya había presentado dos episodios de

Tabla 1. Resumen de los hallazgos más frecuentes en pacientes con esofagitis necrotizante (esófago negro) descritos en la literatura*

\begin{tabular}{|c|c|c|c|c|c|c|}
\hline $\begin{array}{l}\text { Edad- } \\
\text { sexo** }\end{array}$ & Síntomas & $\begin{array}{l}\text { Hallazgos } \\
\text { endoscópicos }\end{array}$ & $\begin{array}{l}\text { Longitud } \\
\text { de la } \\
\text { necrosis }\end{array}$ & $\begin{array}{l}\text { Hallazgos } \\
\text { histológicos }\end{array}$ & Desenlace & Enfermedades de base \\
\hline${ }^{t} n=261$ & $n=148$ & $n=241$ & $n=106$ & $n=39$ & $n=261$ & $\begin{array}{l}n=157 \\
\text { Enf. graves: } 78 \%\end{array}$ \\
\hline 70 años & $\begin{array}{l}\text { Hemorragia } \\
\text { digestiva: } 66 \%\end{array}$ & $\begin{array}{l}\text { Esófago negro: } 91 \% \\
\text { Necrosis: } 10 \%\end{array}$ & $\begin{array}{l}1 / 3: 22,9 \% \\
2 / 3: 40 \%\end{array}$ & $\begin{array}{l}\text { Autopsia: } 8 \\
\text { Necrosis: } 97 \%\end{array}$ & $\begin{array}{l}\text { Curación: } 38 \text { \% } \\
\text { Mejoría: } 5 \%\end{array}$ & $n=104$ \\
\hline $172 / N$ & Dolor: $20 \%$ & Úlcera esofágica: 2,5 \% & $3 / 3: 33 \%$ & Inflamación: 45 \% & Muerte: $32 \%$ & Hemorragia y choque: $36 \%$ \\
\hline 61/M & Hematemesis: $20 \%$ & Úlcera duodenal: 4 \% & $1 / 2: 3,8 \%$ & Isquemia & Estenosis: $10 \%$ & Hipertensión arterial: 27 \% \\
\hline \multirow[t]{11}{*}{$26 / ?$} & Melenas: $16 \%$ & Sangrado activo: 1,7 \% & & Esófago: 24 \% & Residual: 6 \% & Cardiopatía: 24 \% \\
\hline & Vómito: $11 \%$ & Perforación: 1,3 \% & & Estómago: $5 \%$ & Stents: $1 \%$ & Insuficiencia hepática: 24 \% \\
\hline & Melanemesis: $10 \%$ & Úlcera gástrica: 1 \% & & Duodeno: $5 \%$ & Falla multiorgánica: 3 \% & Cetoacidosis diabética: $20 \%$ \\
\hline & Disfagia: $9 \%$ & Estenosis esófago: $1 \%$ & & Úlcera esófago: 16 \% & Esofaguectomía: 1,5 \% & Insuficiencia renal: $18 \%$ \\
\hline & Choque: $7 \%$ & & & Trombosis: $16 \%$ & Neumotórax y & Septicemia: $14 \%$ \\
\hline & Anemia: $4 \%$ & & & Hemorragia: 8 \% & neumomediastino: $1 \%$ & Alcoholismo: $14 \%$ \\
\hline & Muerte súbita: $3 \%$ & & & & Fístula esofágica: 0,3 \% & ${ }^{t+A I N E}: 10 \%$ \\
\hline & Inconciencia: 3 \% & & & & Esófago-gastrostomía: 0,3 \% & Hernia hiatal: $6 \%$ \\
\hline & & & & & Perforación duodenal: 0,3% & Fibrilación auricular: 6 \% \\
\hline & & & & & Isquemia mesentérica: 0,3\% & Neoplasia: $4 \%$ \\
\hline & & & & & Vólvulo gástrico: 0,3 \% & $\begin{array}{l}\text { Secuelas ECV: } 4 \% \\
\text { Amputación: } 4 \%\end{array}$ \\
\hline
\end{tabular}

\footnotetext{
*Consolidado de hallazgos clínicos y desenlaces en todos los casos descritos en la literatura (3, 8-11, 13-81).

** Sexo: V: varón, M: mujer.

${ }^{+} n$ : número de pacientes de los cuales se tiene la descripción del ítem correspondiente

${ }^{+}$AINE: antiinflamatorios no esteroideos.
} 
Tabla 2. Casos descritos en la literatura médica de esofagitis necrotizante (esófago negro)

\begin{tabular}{|c|c|c|c|c|c|c|c|}
\hline $\begin{array}{l}\text { Referencia y } \\
\text { primer autor }\end{array}$ & $\begin{array}{l}\text { Edad- } \\
\text { género }\end{array}$ & Síntoma & $\begin{array}{l}\text { Longitud } \\
\text { de la } \\
\text { necrosis }\end{array}$ & $\begin{array}{c}\text { Hallazgos } \\
\text { endoscópicos }\end{array}$ & $\begin{array}{l}\text { Hallazgos } \\
\text { histológicos }\end{array}$ & $\begin{array}{l}\text { Tratamiento } \\
\text { quirúrgico }\end{array}$ & Enfermedades de base ${ }^{*}$ \\
\hline $\begin{array}{l}\text { 11. Abed J } \\
\text { et al }\end{array}$ & $80 / \mathrm{V}$ & $\begin{array}{l}\text { Dolor difuso de } \\
\text { abdomen, melena }\end{array}$ & $3 / 3$ & $\begin{array}{l}\text { Mucosa ulcerada, negra } \\
\text { y necrótica }\end{array}$ & $\begin{array}{l}\text { Necrosis extensa, } \\
\text { inflamación severa, } \\
\text { ausencia de epitelio }\end{array}$ & Curación & $\begin{array}{l}\text { Colitis ulcerativa, ERGE, } \\
\text { antiácidos y bismuto } \\
\text { subsalicilato }\end{array}$ \\
\hline $\begin{array}{l}\text { 12. Abu-Zaid } \\
\text { A et al }\end{array}$ & $40 / \mathrm{V}$ & & $2 / 3$ & $\begin{array}{l}\text { Esófago negro, mucosa } \\
\text { necrótica, ulceraciones } \\
\text { superficiales múltiples }\end{array}$ & $\begin{array}{l}\text { Infiltrado inflamatorio } \\
\text { focal, ulceración, } \\
\text { necrosis, hemorragia }\end{array}$ & Curación & \\
\hline $\begin{array}{l}13 . \\
\text { Akkinepally S } \\
\text { et al }\end{array}$ & $60 / \mathrm{N}$ & $\begin{array}{l}\text { Inconciencia, } \\
\text { melanemesis y melena }\end{array}$ & $1 / 2$ & $\begin{array}{l}\text { Esófago negro, } \\
\text { gastropatía hipertensiva } \\
\text { grave, erosiones } \\
\text { isquémicas en el } \\
\text { duodeno }\end{array}$ & & $\begin{array}{l}\text { Fallece con síndrome } \\
\text { hepatorrenal y sepsis }\end{array}$ & $\begin{array}{l}\text { Abuso de alcohol, HVDA, } \\
\text { cirrosis e hipotensión }\end{array}$ \\
\hline $\begin{array}{l}14 . \\
\text { Altenburger } \\
\text { DL et al }\end{array}$ & $45 / \mathrm{M}$ & & $3 / 3$ & & $\begin{array}{l}\text { Infiltrado inflamatorio } \\
\text { agudo difuso, } \\
\text { pigmentación por } \\
\text { lipofuscina por } \\
\text { isquemia }\end{array}$ & $\begin{array}{l}\text { Autopsia: esófago negro, } \\
\text { necrosis isquémica } \\
\text { mucosa, submucosa y } \\
\text { muscular }\end{array}$ & Cocaína y alcohol \\
\hline $\begin{array}{l}\text { 15. Ambrosio } \\
\text { MR et al }\end{array}$ & $18 / \mathrm{N}$ & $\begin{array}{l}\text { Síndrome de } \\
\text { choque tóxico tipo } \\
\text { estreptocócico }\end{array}$ & $3 / 3$ & & $\begin{array}{l}\text { Esófago negro } \\
\text { circunferencial total }\end{array}$ & Fallece-autopsia & \\
\hline $\begin{array}{l}\text { 4. Augusto F } \\
\text { et al }\end{array}$ & $\begin{array}{l}23 / \mathrm{N} \\
6 / \mathrm{M} \\
40 \text { a } 91 \\
\text { años, } \\
\text { media } \\
75,2\end{array}$ & $\begin{array}{l}\text { Hematemesis o melenas } \\
\text { Ocho pacientes } \\
\text { con inestabilidad } \\
\text { hemodinámica }\end{array}$ & $\begin{array}{l}2 / 3 \text { en } 17 \\
3 / 3 \text { en } 10 \\
1 / 3 \text { en } 1 \\
29 \text { casos }\end{array}$ & Esófago negro & & $\begin{array}{l}\text { Fallecen } 10 \%->34 \% \\
\text { por enfermedad de base }\end{array}$ & $\begin{array}{l}24(82,3 \%) \text { con enfermedad } \\
\text { de base grave, DM-II e HTA }\end{array}$ \\
\hline $\begin{array}{l}\text { 16. Barnes T } \\
\text { et al }\end{array}$ & $53 / \mathrm{V}$ & $\begin{array}{l}\text { Melanemesis el día } 15 \\
\text { de cefazolina }\end{array}$ & & Esofagitis necrotizante & & Curación & $\begin{array}{l}\text { Septicemia y osteomielitis } \\
\text { femoral por Staphylococcus } \\
\text { aureus. TP de } 89,2 \text { e INR } \\
\text { de } 8,11\end{array}$ \\
\hline $\begin{array}{l}\text { 17. Bremholm } \\
\text { Let al }\end{array}$ & & & $3 / 3$ & Negro difuso & & Fallece a las 48 horas & Hemorragia e hipoperfusión \\
\hline
\end{tabular}


Tabla 2. Casos descritos en la literatura médica de esofagitis necrotizante (esófago negro). Continuación

\begin{tabular}{|c|c|c|c|c|c|c|c|c|}
\hline $\begin{array}{l}\text { Referencia y } \\
\text { primer autor }\end{array}$ & $\begin{array}{l}\text { Edad- } \\
\text { género }\end{array}$ & Síntoma & $\begin{array}{l}\text { Longitud } \\
\text { de la } \\
\text { necrosis }\end{array}$ & $\begin{array}{c}\text { Hallazgos } \\
\text { endoscópicos }\end{array}$ & $\begin{array}{l}\text { Hallazgos } \\
\text { histológicos }\end{array}$ & Desenlace & $\begin{array}{l}\text { Tratamiento } \\
\text { quirúrgico }\end{array}$ & Enfermedades de base* \\
\hline $\begin{array}{l}\text { 18. Cameron } \\
\text { PA, } \\
\text { Schweiger } F\end{array}$ & $62 / \mathrm{V}$ & $\begin{array}{l}\text { Inconciencia, } \\
\text { hipovolemia, hipotermia, } \\
\text { insuficiencia renal } \\
\text { aguda, SNG* } \\
\text { Cuncho de café }\end{array}$ & $3 / 3$ & Esófago negro & $\begin{array}{l}\text { Necrosis esofágica } \\
\text { aguda y pigmento } \\
\text { marrón difuso }\end{array}$ & Curación & & $\begin{array}{l}\text { Colangitis esclerosante. } \\
\text { Enfermedad coronaria, HTA, } \\
\text { DM-II, alcoholismo }\end{array}$ \\
\hline $\begin{array}{l}\text { 19. Caravaca- } \\
\text { Fontán F et al }\end{array}$ & $65 / V$ & $\begin{array}{l}\text { Dispepsia, náuseas, } \\
\text { ardor retroesternal y } \\
\text { melanemesis }\end{array}$ & $3 / 3$ & $\begin{array}{l}\text { Esófago negro } \\
\text { circunferencial difuso }\end{array}$ & & Curación & & $\begin{array}{l}24 \text { h postrasplante renal, } \\
\text { edema pulmonar y paro } \\
\text { cardíaco, estenosis aórtica, } \\
\text { balón aórtico y reemplazo } \\
\text { valvular transcatéter }\end{array}$ \\
\hline $\begin{array}{l}\text { 20. Castaño- } \\
\text { Llano R et al }\end{array}$ & $\begin{array}{l}68,69 \\
\text { y } 82 \\
\text { años/M }\end{array}$ & Hematemesis & $\begin{array}{l}2 / 3 \\
3 \text { casos }\end{array}$ & Esófago negro & $\begin{array}{l}\text { Una biopsia con } \\
\text { isquemia }\end{array}$ & $\begin{array}{l}\text { Dos mejoran } \\
\text { Una Fallece en falla } \\
\text { multiorgánica }\end{array}$ & & $\begin{array}{l}\text { Cirrosis hepática / } \\
\text { mastectomía por CA de seno } \\
\text { / metástasis hígado }\end{array}$ \\
\hline $\begin{array}{l}\text { 21. Choi EJ } \\
\text { et al }\end{array}$ & $48 / \mathrm{V}$ & Hematemesis & $3 / 3$ & $\begin{array}{l}\text { Esófago negro difuso, } \\
\text { friable }\end{array}$ & $\begin{array}{l}\text { Infiltración } \\
\text { inflamatoria, tejido } \\
\text { necrótico }\end{array}$ & Curación & & \\
\hline $\begin{array}{l}\text { 3. Day A, } \\
\text { Sayegh M }\end{array}$ & $\begin{array}{l}\text { 68,4 años } \\
(19-91) \\
88 / \mathrm{N} \\
22 / \mathrm{M}, 2 ?\end{array}$ & $\begin{array}{l}\text { HVDA } 65 \% \text {, dolor } \\
\text { epigástrico } 8 \% \text {, disfagia } \\
7 \% \text {, melena } 5 \%\end{array}$ & $\begin{array}{l}112 \\
\text { casos } 1 / 3 \\
\text { a } 2 / 3 \text { la } \\
\text { mayoría }\end{array}$ & $\begin{array}{l}\text { Esófago negro, friable, } \\
\text { exudados amarillos y } \\
\text { ulceración, demarcación } \\
\text { clara cardial }\end{array}$ & $\begin{array}{l}\text { Necrosis severa } \\
\text { mucosa y submucosa } \\
\text { e inflamación de las } \\
\text { fibras musculares }\end{array}$ & $\begin{array}{l}\text { Muerte: } 38,4 \% \\
\text { Resolución: } 49,1 \% \\
\text { Estenosis: } 7,1 \% \\
\text { Desconocido: } 3,6 \% \\
\text { Otros: } 1,8 \%\end{array}$ & & $\begin{array}{l}\text { Choque / hipotensión: } \\
20 \% \text {; falla hepática: } 15 \% \text {; } \\
\text { neoplasia: } 11 \% \text {; infección: } \\
7 \% \\
\text { Desconocido: } 26 \% \\
\text { Otros: } 21 \%\end{array}$ \\
\hline $\begin{array}{l}\text { 22. De Palma } \\
\text { GD et al }\end{array}$ & $82 / \mathrm{N}$ & Melanemesis & $3 / 3$ & $\begin{array}{l}\text { Esófago negro } \\
\text { circunferencial }\end{array}$ & & & & $\begin{array}{l}\text { Enfermedad coronaria, HTA, } \\
\text { DM }\end{array}$ \\
\hline \multirow[t]{2}{*}{$\begin{array}{l}\text { 23. Edling } P \text {, } \\
\text { Thomsen } \mathrm{H}\end{array}$} & & Hematemesis & & $\begin{array}{l}\text { Necrosis esofágica } \\
\text { aguda }\end{array}$ & & Curación & & Cetoacidosis diabética \\
\hline & & Hematemesis & & $\begin{array}{l}\text { Necrosis esofágica } \\
\text { aguda }\end{array}$ & & Curación & & Cetoacidosis diabética \\
\hline $\begin{array}{l}\text { 24. Eren B } \\
\text { et al }\end{array}$ & $17 / M$ & $\begin{array}{l}\text { Distensión abdominal y } \\
\text { vómito }\end{array}$ & & & & $\begin{array}{l}\text { Fallece a } 6 \text { horas, } \\
\text { autopsia con esófago } \\
\text { negro }\end{array}$ & & \\
\hline $\begin{array}{l}25 . \\
\text { Fernández R } \\
\text { et al }\end{array}$ & $87 / M$ & $\begin{array}{l}\text { HVDA luego de } \\
\text { anestesia espinal }\end{array}$ & & $\begin{array}{l}\text { Esófago negro con } \\
\text { sangrado activo }\end{array}$ & & Fallece & & HTA, fibrilación auricular \\
\hline
\end{tabular}


Tabla 2. Casos descritos en la literatura médica de esofagitis necrotizante (esófago negro). Continuación

\begin{tabular}{|c|c|c|c|c|c|c|c|c|}
\hline $\begin{array}{l}\text { Referencia y } \\
\text { primer autor }\end{array}$ & $\begin{array}{l}\text { Edad- } \\
\text { género }\end{array}$ & Síntoma & $\begin{array}{l}\text { Longitud } \\
\text { de la } \\
\text { necrosis }\end{array}$ & $\begin{array}{c}\text { Hallazgos } \\
\text { endoscópicos }\end{array}$ & $\begin{array}{l}\text { Hallazgos } \\
\text { histológicos }\end{array}$ & Desenlace & $\begin{array}{l}\text { Tratamiento } \\
\text { quirúrgico }\end{array}$ & Enfermedades de base ${ }^{*}$ \\
\hline $\begin{array}{l}26 . \\
\text { Fernández- } \\
\text { Carrillo C } \\
\text { et al }\end{array}$ & $94 / \mathrm{V}$ & Melanemesis & $1 / 3$ & $\begin{array}{l}\text { Placas necróticas } \\
\text { distales }\end{array}$ & & Curación & & $\begin{array}{l}\text { Angina de pecho, hipotensión } \\
\text { arterial y fractura femoral, con } \\
\text { fibrilación auricular crónica }\end{array}$ \\
\hline $\begin{array}{l}27 . \\
\text { Galanopou- } \\
\text { los M et al }\end{array}$ & $80 / \mathrm{M}$ & Melena & $1 / 2$ & $\begin{array}{l}\text { Esófago negro } \\
\text { circunferencial }\end{array}$ & $\begin{array}{l}\text { Necrosis epitelial, con } \\
\text { exudado inflamatorio } \\
\text { inespecífico }\end{array}$ & Curación & & $\begin{array}{l}\text { Ibuprofeno } 1600 \mathrm{mg} / \mathrm{d}, 1 \\
\text { semana y anemia ferropénica } \\
(\mathrm{Hb} 9 \mathrm{~g} \%)\end{array}$ \\
\hline $\begin{array}{l}\text { 28. Galtés I } \\
\text { et al }\end{array}$ & $54 / \mathrm{V}$ & Inconciencia & $3 / 3$ & $\begin{array}{l}\text { Necrosis esofágica } \\
\text { aguda }\end{array}$ & & Fallece & & $\begin{array}{l}\text { Alcoholismo, politoxicomanía, } \\
\text { DM-I }\end{array}$ \\
\hline $\begin{array}{l}\text { 29. Garas G } \\
\text { et al }\end{array}$ & $89 / \mathrm{M}$ & $\begin{array}{l}\text { Epigastralgia, náuseas, } \\
\text { vómito, hematemesis y } \\
\text { melenas }\end{array}$ & $2 / 3$ & & $\begin{array}{l}\text { Isquemia, necrosis } \\
\text { hemorrágica }\end{array}$ & FMO, fallece & $\begin{array}{l}\text { Hernia hiatal } \\
\text { grande, vólvulo } \\
\text { gástrico }\end{array}$ & AAA y cayado + trombo mural \\
\hline $\begin{array}{l}\text { 30. Gómez } \\
\text { AA et al }\end{array}$ & $67 / N$ & & & Esófago negro & & $\begin{array}{l}\text { Estenosis + prótesis } \\
\text { autoexpandible }\end{array}$ & & $\begin{array}{l}\text { DM y cetoacidosis, fibrilación } \\
\text { auricular, insuficiencia renal } \\
\text { crónica, sepsis grave }\end{array}$ \\
\hline \multirow[t]{7}{*}{$\begin{array}{l}\text { 10. Gómez LJ } \\
\text { et al }\end{array}$} & $82 / N$ & $\begin{array}{l}\text { Hematemesis, } \\
\text { epigastralgia }\end{array}$ & $3 / 3$ & Esófago negro & $\begin{array}{l}\text { Erosión o ulceración, } \\
\text { necrosis extensa, } \\
\text { trombosis vascular en } \\
5 \text { de } 7\end{array}$ & Curación & & $\begin{array}{l}\text { Cardiopatía, valvulopatía, } \\
\text { fibrilación auricular crónica, } \\
\text { warfarina }\end{array}$ \\
\hline & $87 / M$ & $\begin{array}{l}\text { Hematemesis y } \\
\text { rectorragia }\end{array}$ & $2 / 3$ & Esófago negro & & Curación & & $\begin{array}{l}\text { DM, HTA, fibrilación auricular, } \\
\text { Warfarina }\end{array}$ \\
\hline & $71 / \mathrm{M}$ & $\begin{array}{l}\text { Melanemesis, vómito, } \\
\text { epigastralgia }\end{array}$ & $3 / 3$ & Esófago negro & & Fallece, FMO & & $\begin{array}{l}\text { Cardiopatía, DM y } \\
\text { cetoacidosis }\end{array}$ \\
\hline & $77 / \mathrm{N}$ & $\begin{array}{l}\text { Melanemesis, vómito, } \\
\text { epigastralgia }\end{array}$ & $2 / 3$ & $\begin{array}{l}\text { Esófago negro más } \\
\text { úlcera duodenal }\end{array}$ & & Curación & & Insuficiencia renal \\
\hline & $83 / \mathrm{V}$ & Hematemesis & $3 / 3$ & Esófago negro & & Curación & & Insuficiencia renal \\
\hline & $63 / \mathrm{N}$ & $\begin{array}{l}\text { Melanemesis, vómito, } \\
\text { dispepsia }\end{array}$ & $2 / 3$ & $\begin{array}{l}\text { Esófago negro más } 2 \\
\text { úlceras duodenales }\end{array}$ & & Curación & & EPOC \\
\hline & $94 / \mathrm{M}$ & Melanemesis, vómito & $3 / 3$ & Esófago negro & & $\begin{array}{l}\text { Fallece día } 28 \text { FMO y } \\
\text { sepsis }\end{array}$ & & $\begin{array}{l}\text { HH, esofagitis G-IV, } \\
\text { insuficiencia renal }\end{array}$ \\
\hline
\end{tabular}


Tabla 2. Casos descritos en la literatura médica de esofagitis necrotizante (esófago negro). Continuación

\begin{tabular}{|c|c|c|c|c|c|c|c|c|}
\hline $\begin{array}{l}\text { Referencia y } \\
\text { primer autor }\end{array}$ & $\begin{array}{l}\text { Edad- } \\
\text { género }\end{array}$ & Síntoma & $\begin{array}{l}\text { Longitud } \\
\text { de la } \\
\text { necrosis }\end{array}$ & $\begin{array}{c}\text { Hallazgos } \\
\text { endoscópicos }\end{array}$ & $\begin{array}{l}\text { Hallazgos } \\
\text { histológicos }\end{array}$ & Desenlace & $\begin{array}{l}\text { Tratamiento } \\
\text { quirúrgico }\end{array}$ & Enfermedades de base ${ }^{*}$ \\
\hline $\begin{array}{l}\text { 31. Gómez V } \\
\text { et al }\end{array}$ & $50 / M$ & Disfagia & $3 / 3$ & $\begin{array}{l}\text { Esófago coloración } \\
\text { grisácea y negra } \\
\text { difusamente }\end{array}$ & $\begin{array}{l}\text { Necrosis esofágica } \\
\text { aguda con células } \\
\text { inflamatorias } \\
\text { dispersas con } \\
\text { bacterias y levaduras }\end{array}$ & $\begin{array}{l}\text { Ulceración profunda } \\
\text { difusa y estenosis distal } \\
\text { de } 3 \mathrm{~mm}, 1 / 2 \text { inferior } \\
\text { a las } 10 \text { semanas. } \\
\text { Dilataciones sin } \\
\text { resultado }\end{array}$ & $\begin{array}{l}\text { Stent } \\
\text { autoexpandible, } \\
\text { fístula tercio } \\
\text { medio, } \\
\text { esofaguectomía }\end{array}$ & $\begin{array}{l}42 \text { días postrasplante } \\
\text { hepático, } 10 \text { días posruptura } \\
\text { arteria frénica inferior con } \\
\text { choque hemorrágico }\end{array}$ \\
\hline $\begin{array}{l}\text { 32. Groenveld } \\
\text { RL et al }\end{array}$ & $68 / M$ & FMO, perforación & $1 / 3$ & $\begin{array}{l}\text { Esófago negro } \\
\text { circunferencial, } \\
\text { perforación a } 32 \mathrm{~cm}\end{array}$ & & Estenosis + dilataciones & $\begin{array}{l}\text { Drenaje } \\
\text { neumomediastino } \\
\text { y neumotórax }\end{array}$ & \\
\hline $\begin{array}{l}\text { 33. Gurvits } \\
\text { GE et al }\end{array}$ & $75 / \mathrm{N}$ & 7/8 pacientes con HVDA & $\begin{array}{l}8 \\
\text { pacientes } \\
1 / 3\end{array}$ & $\begin{array}{l}\text { Esofágico distal; } \\
\text { algunos, extensión } \\
\text { proximal. Compromiso } \\
\text { duodenal en el } 50 \text { \% de } \\
\text { los casos }\end{array}$ & & $\begin{array}{l}\text { Estenosis en } 2 / 8 \\
\text { pacientes, fallece } 1 / 8 \text { por } \\
\text { enfermedad de base }\end{array}$ & & $\begin{array}{l}\text { Abuso alcohol, HTA, DM } \\
\text { en } 7 / 8 \text {, dislipidemia, DNT } \\
\text { anemia } 7 / 8 \text {, vasculopatía, } \\
\text { hipoalbuminemia 8/8, IRC 7/8 }\end{array}$ \\
\hline $\begin{array}{l}\text { 34. Hejna P } \\
\text { et al }\end{array}$ & $53 / \mathrm{M}$ & Hallada inconsciente & $3 / 3$ & & $\begin{array}{l}\text { Autopsia: necrosis } \\
\text { epitelial completa, } \\
\text { focal de muscular } \\
\text { mucosa, infiltrado } \\
\text { denso leucocitos y } \\
\text { ulceración }\end{array}$ & Fallece & & $\begin{array}{l}\text { Suicidio con antipsicóticos: } \\
\text { haloperidol, zotepina y } \\
\text { cloroprotixeno }\end{array}$ \\
\hline $\begin{array}{l}\text { 35. Hermet A } \\
\text { et al }\end{array}$ & $72 / N$ & $\begin{array}{l}\text { Hematemesis y } \\
\text { epigastralgia 1. }{ }^{\text {er }} \text { día } \\
\text { postoperatorio }\end{array}$ & & Esófago negro & & Curación & & $\begin{array}{l}\text { Reemplazo de cadera con } \\
\text { compromiso hemodinámico. } \\
\text { Coronariopatía, vasculopatía } \\
\text { MM II, DM, HTA }\end{array}$ \\
\hline $\begin{array}{l}\text { 36. Hong JW } \\
\text { et al }\end{array}$ & $85 / \mathrm{V}$ & $\begin{array}{l}\text { Abuso de alcohol }>100 \\
\text { gramos y hematemesis }\end{array}$ & $2 / 3$ & $\begin{array}{l}\text { Exudados blanquecinos } \\
\text { sugestivos de } \\
\text { candidiasis, } 2 / 3 \text { distales } \\
\text { mucosa negra, edema } \\
\text { y múltiples erosiones } \\
\text { bulbo duodenal }\end{array}$ & $\begin{array}{l}\text { Necrosis epitelial con } \\
\text { exudado inflamatorio } \\
\text { y ulceración. No } \\
\text { cándida }\end{array}$ & Curación & & $\begin{array}{l}\text { HTA, DM, abuso de alcohol, } \\
\text { úlcera gástrica y secuelas } \\
\text { ECV }\end{array}$ \\
\hline $\begin{array}{l}\text { 37. lorio } \mathrm{N} \\
\text { et al }\end{array}$ & $63 / M$ & $\begin{array}{l}\text { Dolor abdominal, } \\
\text { diarrea, náuseas, } \\
\text { vómitos. Púrpura } \\
\text { palpable en } \\
\text { extremidades }\end{array}$ & $3 / 3$ & $\begin{array}{l}\text { Panesofagitis necrótica } \\
\text { ulcerada, duodenitis } \\
\text { necrótica hasta tercera } \\
\text { porción }\end{array}$ & $\begin{array}{l}\text { Granulación tisular } \\
\text { por ulceración. } \\
\text { Vasculitis isquémica } \\
\text { duodenal de mucosa } \\
\text { y submucosa }\end{array}$ & Curación & & $\begin{array}{l}\text { Trasplante renal, DM-II, } \\
\text { enfermedad arterial periférica, } \\
\text { GMN y lesión tubular por } \\
\text { púrpura de Henoch-Schönlein }\end{array}$ \\
\hline
\end{tabular}


Tabla 2. Casos descritos en la literatura médica de esofagitis necrotizante (esófago negro). Continuación

\begin{tabular}{|c|c|c|c|c|c|c|c|c|}
\hline $\begin{array}{l}\text { Referencia y } \\
\text { primer autor }\end{array}$ & $\begin{array}{l}\text { Edad- } \\
\text { género }\end{array}$ & Síntoma & $\begin{array}{l}\text { Longitud } \\
\text { de la } \\
\text { necrosis }\end{array}$ & $\begin{array}{l}\text { Hallazgos } \\
\text { endoscópicos }\end{array}$ & $\begin{array}{l}\text { Hallazgos } \\
\text { histológicos }\end{array}$ & Desenlace & $\begin{array}{l}\text { Tratamiento } \\
\text { quirúrgico }\end{array}$ & Enfermedades de base ${ }^{*}$ \\
\hline $\begin{array}{l}\text { 38. Ishibashi } \\
\text { Y et al }\end{array}$ & $\begin{array}{l}4 \\
\text { pacientes }\end{array}$ & HVDA & $3 / 3$ & Esófago negro & & $\begin{array}{l}\text { Curación en tres } \\
\text { Fallece uno }\end{array}$ & & $\begin{array}{l}\text { Cetoacidosis en tres. DM } \\
\text { en dos }\end{array}$ \\
\hline $\begin{array}{l}39 . \\
\text { Kanaparthi C } \\
\text { et al }\end{array}$ & $76 / \mathrm{M}$ & $\begin{array}{l}\text { Náuseas, dolor } \\
\text { epigástrico, } \\
\text { melanemesis y melena }\end{array}$ & $2 / 3$ & $\begin{array}{l}\text { Esófago negro con } \\
\text { necrosis circunferencial } \\
\text { y ulceración. Múltiples } \\
\text { úlceras gástricas y } \\
\text { duodenales con necrosis }\end{array}$ & $\begin{array}{l}\text { Infiltrado inflamatorio, } \\
\text { necrosis, exudado, } \\
\text { inflamación aguda y } \\
\text { crónica duodeno }\end{array}$ & Curación & & $\begin{array}{l}\text { Coronariopatía, ICC, ECV, } \\
\text { DM-II, HTA, asma y demencia }\end{array}$ \\
\hline $\begin{array}{l}40 . \\
\text { Karaahmet F } \\
\text { et al }\end{array}$ & $81 / \mathrm{M}$ & Hematemesis & $3 / 3$ & $\begin{array}{l}\text { Esófago negro extenso } \\
\text { y difuso, con mucosa } \\
\text { friable }\end{array}$ & & Mejoría & & $\begin{array}{l}\text { HTA, DM-II, insuficiencia } \\
\text { cardíaca, cáncer de colon }\end{array}$ \\
\hline $\begin{array}{l}\text { 41. Kim IK } \\
\text { et al }\end{array}$ & $34 / \mathrm{N}$ & Hematemesis & $2 / 3$ & $\begin{array}{l}\text { Esófago negro, friable, } \\
\text { de } 3 \text { a } 4 \mathrm{~cm} \text { por debajo } \\
\text { del cricofaríngeo al } \\
\text { cardias }\end{array}$ & $\begin{array}{l}\text { Al } 6 .^{\circ} \text { día: necrosis } \\
\text { de submucosa y } \\
\text { candidiasis }\end{array}$ & $\begin{array}{l}\text { Estenosis esofágica a } 2 \\
\text { meses, } 3 \text { dilataciones, } \\
\text { esofaguectomía subtotal } \\
\text { y esofagogastrostomía }\end{array}$ & & $\begin{array}{l}\text { Cetoacidosis diabética con } \\
\text { hipotensión, hiponatremia, } \\
\text { hiperpotasemia, IRA prerrenal }\end{array}$ \\
\hline $\begin{array}{l}\text { 42. Kim YH, } \\
\text { Choi SY }\end{array}$ & $53 / \mathrm{V}$ & Hematemesis & $2 / 3$ & $\begin{array}{l}\text { Esófago negro hasta la } \\
\text { unión gastroesofágica }\end{array}$ & & Mejoría & & \\
\hline $\begin{array}{l}\text { 43. Köksal AS } \\
\text { et al }\end{array}$ & & & & Esófago negro & & Curación & $\begin{array}{l}\text { Perforación } \\
\text { úlcera duodenal }\end{array}$ & \\
\hline $\begin{array}{l}\text { 44. Kwon H-J } \\
\text { et al }\end{array}$ & $67 / M$ & Hematemesis & $2 / 3$ & $\begin{array}{l}\text { Esófago negro, mucosa } \\
\text { macerada, necrosis } \\
\text { circunferencial distal con } \\
\text { coágulo adherido }\end{array}$ & & Curación & & $\begin{array}{l}\text { Disección coronaria arterial } \\
\text { durante cateterismo con } \\
\text { hipotensión aguda }\end{array}$ \\
\hline $\begin{array}{l}\text { 45. Lahbabi } \\
\text { M et al }\end{array}$ & $60 / \mathrm{V}$ & $\begin{array}{l}\text { Hematemesis, choque } \\
\text { séptico }\end{array}$ & $2 / 3$ & $\begin{array}{l}\text { Esófago negro } \\
\text { circunferencial con } \\
\text { exudado y sangrado } \\
\text { difuso }\end{array}$ & $\begin{array}{l}\text { Respuesta } \\
\text { inflamatoria local, } \\
\text { necrosis de mucosa y } \\
\text { submucosa }\end{array}$ & $\begin{array}{l}\text { Fallece a } 6 \text { horas por } \\
\text { choque séptico }\end{array}$ & & $\begin{array}{l}\text { Necrosis isquémica y } \\
\text { supurativa del muñón }\end{array}$ \\
\hline $\begin{array}{l}\text { 46. Lin MC } \\
\text { et al }\end{array}$ & $70 / \mathrm{V}$ & $\begin{array}{l}\text { Melena y hematemesis } \\
\text { por } 2 \text { días }\end{array}$ & $2 / 3$ & $\begin{array}{l}\text { Esófago negro y } \\
\text { ulceración extensa }\end{array}$ & & Curación & & $\begin{array}{l}\text { HTA, DM, IRC, fibrilación } \\
\text { auricular, síndrome del seno } \\
\text { enfermo y cáncer de próstata }\end{array}$ \\
\hline $\begin{array}{l}\text { 47. Maher } \\
\text { MM, Nassar } \\
\text { Ml }\end{array}$ & $63 / \mathrm{V}$ & Melanemesis & $2 / 3$ & Mucosa negra y friable & $\begin{array}{l}\text { Necrosis focal, } \\
\text { hialinosis de } \\
\text { pequeños vasos }\end{array}$ & Fallece & & $\begin{array}{l}\text { DM-II, HTA, EPOC, SDRA, } \\
\text { IRA, choque }\end{array}$ \\
\hline
\end{tabular}


Tabla 2. Casos descritos en la literatura médica de esofagitis necrotizante (esófago negro). Continuación

\begin{tabular}{|c|c|c|c|c|c|c|c|c|}
\hline $\begin{array}{l}\text { Referencia y } \\
\text { primer autor }\end{array}$ & $\begin{array}{l}\text { Edad- } \\
\text { género }\end{array}$ & Síntoma & $\begin{array}{l}\text { Longitud } \\
\text { de la } \\
\text { necrosis }\end{array}$ & $\begin{array}{c}\text { Hallazgos } \\
\text { endoscópicos }\end{array}$ & $\begin{array}{l}\text { Hallazgos } \\
\text { histológicos }\end{array}$ & Desenlace & $\begin{array}{l}\text { Tratamiento } \\
\text { quirúrgico }\end{array}$ & Enfermedades de base ${ }^{*}$ \\
\hline 48. Maroy B & $81 / \mathrm{V}$ & Hematemesis & $1 / 2$ & $\begin{array}{l}\text { Várices grado } 3 \text { con } \\
\text { mucosa negra y necrosis } \\
\text { entre } 30 \text { y } 40 \mathrm{~cm} . \\
\text { Ligadura }\end{array}$ & & Curación & & $\begin{array}{l}\text { Hepatectomía derecha y } \\
\text { segmento IV por hepatoma } \\
\text { y enfermedad venooclusiva } \\
\text { de segmentos II y III por } \\
\text { I }^{131} \text {-Lipiodol }\end{array}$ \\
\hline $\begin{array}{l}49 . \\
\text { McLaughlin } \\
\text { CW et al }\end{array}$ & $62 / M$ & $\begin{array}{l}\text { Náuseas, melanemesis, } \\
\text { dolor retroesternal }\end{array}$ & $1 / 2$ & Esófago necrótico distal & & Curación & & $\begin{array}{l}\text { Reflujo gastroesofágico y } \\
\text { hernia hiatal grande }\end{array}$ \\
\hline $\begin{array}{l}\text { 50. Messner } \\
Z \text { et al }\end{array}$ & $45 / \mathrm{V}$ & Melena, dolor abdominal & $1 / 3$ & $\begin{array}{l}\text { Esófago negro con } \\
\text { sangrado masivo }\end{array}$ & & Mejoría & $\begin{array}{l}\text { Endoprótesis } \\
\text { Ella-Danis }\end{array}$ & \\
\hline $\begin{array}{l}\text { 51. Nikolić S, } \\
\text { Zivković V }\end{array}$ & $76 / \mathrm{V}$ & Muerte súbita & & & $\begin{array}{l}\text { Autopsia: esofagitis } \\
\text { aguda necrotizante. } \\
\text { Erosiones agudas } \\
\text { gastroduodenales }\end{array}$ & Fallece por HVDA & & ECV antiguo-inmovilidad \\
\hline $\begin{array}{l}52 . \\
\text { Pastuszak M, } \\
\text { Groszewski K }\end{array}$ & $87 / M$ & $\begin{array}{l}\text { Hemorragia digestiva } \\
\text { alta, hipotensión arterial }\end{array}$ & & Esófago negro & & Curación & & $\begin{array}{l}\text { Trastornos hemodinámicos, } \\
\text { síntomas de RGE }\end{array}$ \\
\hline $\begin{array}{l}\text { 53. Pereira } 0 \\
\text { et al }\end{array}$ & $62 / M$ & $\begin{array}{l}\text { Melena, anemia aguda } \\
8,4 \mathrm{~g}\end{array}$ & $2 / 3$ & $\begin{array}{l}\text { Candidiasis extensa, } \\
\text { esófago negro }\end{array}$ & & $\begin{array}{l}\text { Estenosis } 1 / 3 \text { distal, } \\
\text { dilataciones y curación }\end{array}$ & & $\begin{array}{l}\text { Hepatitis alcohólica, DHT, } \\
\text { hipotensión arterial, DNT, } \\
\text { anemia por deficiencia de } \\
\text { folato }\end{array}$ \\
\hline $\begin{array}{l}\text { 54. Pierini A } \\
\text { et al }\end{array}$ & & & & Esófago negro & & Curación & & \\
\hline $\begin{array}{l}\text { 55. Plaza- } \\
\text { Santos R } \\
\text { et al }\end{array}$ & $47 \mathrm{~N}$ & Hematemesis por 3 días & $2 / 3$ & $\begin{array}{l}\text { 2/3 mucosa } \\
\text { ulcerada, necrótica, } \\
\text { circunferencial, con } \\
\text { exudado de fibrina }\end{array}$ & & Mejoría & & $\begin{array}{l}\text { Cetoacidosis diabética, IRA, } \\
\text { alcohólico bebedor }>100 \mathrm{~g} / \mathrm{d}\end{array}$ \\
\hline $\begin{array}{l}\text { 56. Pramparo } \\
\text { SE et al }\end{array}$ & $80 / N$ & $\begin{array}{l}\text { Disfagia, hipo, } \\
\text { melanemesis }\end{array}$ & $2 / 3$ & Necrosis esofágica & Necrosis isquémica & Fallece & & $\begin{array}{l}\text { DM-II, HTA. enfermedad } \\
\text { vascular periférica, reemplazo } \\
\text { valvular aórtico, tabaquismo }\end{array}$ \\
\hline
\end{tabular}


Tabla 2. Casos descritos en la literatura médica de esofagitis necrotizante (esófago negro). Continuación

\begin{tabular}{|c|c|c|c|c|c|c|c|}
\hline $\begin{array}{l}\text { Referencia y } \\
\text { primer autor }\end{array}$ & $\begin{array}{l}\text { Edad- } \\
\text { género }\end{array}$ & Síntoma & $\begin{array}{l}\text { Longitud } \\
\text { de la } \\
\text { necrosis }\end{array}$ & $\begin{array}{c}\text { Hallazgos } \\
\text { endoscópicos }\end{array}$ & $\begin{array}{l}\text { Hallazgos } \\
\text { histológicos }\end{array}$ & $\begin{array}{l}\text { Tratamiento } \\
\text { quirúrgico }\end{array}$ & Enfermedades de base* \\
\hline $\begin{array}{l}\text { 9. Ramos R } \\
\text { et al }\end{array}$ & $\begin{array}{l}68 \text { a } 87 \\
\text { años, } \\
\text { media } \\
76,2 \\
9 / \mathrm{N}, 2 / \mathrm{M}\end{array}$ & $\begin{array}{l}\text { Melenas, hematemesis, } \\
\text { vómitos, dolor } \\
\text { abdominal }\end{array}$ & $\begin{array}{l}1 / 3 \text { a } 3 / 3 \\
11 \text { casos }\end{array}$ & Esófago negro & $\begin{array}{l}6 \text { biopsias: necrosis } \\
\text { de mucosa y } \\
\text { submucosa }\end{array}$ & $\begin{array}{l}\text { Siete fallecen } \\
\text { Cuatro mejoran }\end{array}$ & $\begin{array}{l}\text { Nueve con ICC } \\
\text { Dos con cirrosis hepáticas }\end{array}$ \\
\hline $\begin{array}{l}\text { 57. Rigolon R } \\
\text { et al }\end{array}$ & $50 / \mathrm{N}$ & $\begin{array}{l}\text { Disfagia, vómitos, fiebre, } \\
\text { dolor epigástrico }\end{array}$ & $2 / 3$ & $\begin{array}{l}\text { Necrosis circunferencial } \\
\text { de } 24 \text { a } 40 \mathrm{~cm} \text { de la } \\
\text { arcada dentaria }\end{array}$ & $\begin{array}{l}\text { Necrosis tisular, } \\
\text { tejido de granulación, } \\
\text { infiltración leucocitaria } \\
\text { y hemosiderosis }\end{array}$ & $\begin{array}{l}\text { Disfagia, estenosis distal } \\
\text { de } 10 \mathrm{~cm} \text { por } 1 \mathrm{~cm}, 3 \\
\text { dilataciones, curación }\end{array}$ & $\begin{array}{l}\text { HTA, cetoacidosis diabética, } \\
\text { deshidratación, con } \\
\text { hipotensión y taquicardia al } \\
\text { ingreso }\end{array}$ \\
\hline $\begin{array}{l}\text { 58. Rodrigo } \\
\text { M et al }\end{array}$ & $77 / \mathrm{N}$ & Hematemesis & $2 / 3$ & $\begin{array}{l}\text { Necropsia: esofagitis } \\
\text { aguda necrotizante, } \\
\text { aneurisma aorta roto }\end{array}$ & $\begin{array}{l}\text { Mucosa isquémica } \\
\text { desde los } 30 \mathrm{~cm} \text { y } \\
\text { necrótico hasta los } \\
38 \mathrm{~cm}\end{array}$ & & Uso de AINE \\
\hline $\begin{array}{l}\text { 59. Román- } \\
\text { Fernández A } \\
\text { et al }\end{array}$ & & $\begin{array}{l}\text { Hemorragia masiva, } \\
\text { hipotensión arterial }\end{array}$ & & Esófago negro & & & $\begin{array}{l}\text { Anestesia subaracnoidea } \\
\text { para artroplastia parcial de } \\
\text { cadera }\end{array}$ \\
\hline $\begin{array}{l}\text { 60. Salem GA } \\
\text { et al }\end{array}$ & $62 / \mathrm{M}$ & $\begin{array}{l}\text { Hematemesis, } \\
\text { hipotermia }\end{array}$ & & $\begin{array}{l}\text { Necrosis esofágica } \\
\text { aguda }\end{array}$ & & Curación & Alcoholismo \\
\hline $\begin{array}{l}\text { 61. Santos } \\
\text { VM et al }\end{array}$ & $83 / \mathrm{N}$ & $\begin{array}{l}\text { Vómitos, disfagia, } \\
\text { pérdida de peso }\end{array}$ & $1 / 3$ & $\begin{array}{l}\text { Esófago distal con } \\
\text { estenosis, úlcera, } \\
\text { coloración negra }\end{array}$ & $\begin{array}{l}\text { Esofagitis aguda } \\
\text { necrotizante }\end{array}$ & Mejoría & DM-II, HTA, cáncer rectal \\
\hline $\begin{array}{l}\text { 62. Shafa S } \\
\text { et al }\end{array}$ & $62 / \mathrm{N}$ & $\begin{array}{l}\text { Hipotensión e hipoxia a } \\
\text { pesar intubación }\end{array}$ & $3 / 3$ & $\begin{array}{l}\text { Esófago negro total, } \\
\text { perforación grande a } \\
35 \mathrm{~cm}\end{array}$ & & Fallece & $\begin{array}{l}\text { Amputación por fascitis } \\
\text { necrotizante, neumotórax } \\
\text { grande derecho POP por } \\
\text { sonda en pleura derecha }\end{array}$ \\
\hline & $63 / \mathrm{N}$ & $\begin{array}{l}\text { Dolor torácico agudo, } \\
\text { náuseas hematemesis }\end{array}$ & $3 / 3$ & $\begin{array}{l}\text { Necrosis esofágica } \\
\text { difusa, exfoliación } \\
\text { parcial, sangrado } \\
\text { continuo, hemostasia } \\
\text { con epinefrina }\end{array}$ & & Mejoría & ICC, EPOC, abuso de alcohol \\
\hline & $72 / \mathrm{N}$ & $\begin{array}{l}2 .^{\circ} \mathrm{DPO} \text { melena y baja } \\
2 \mathrm{~g} \mathrm{Hb}\end{array}$ & $3 / 3$ & $\begin{array}{l}\text { Necrosis esofágica } \\
\text { aguda difusa }\end{array}$ & $\begin{array}{l}\text { Exudado inflamatorio } \\
\text { y tejido necrótico }\end{array}$ & Curación & $\begin{array}{l}\text { Amputación bilateral } \\
\text { subrotuliana, con hemorragia }\end{array}$ \\
\hline
\end{tabular}


Tabla 2. Casos descritos en la literatura médica de esofagitis necrotizante (esófago negro). Continuación

\begin{tabular}{|c|c|c|c|c|c|c|c|c|}
\hline $\begin{array}{l}\text { Referencia y } \\
\text { primer autor }\end{array}$ & $\begin{array}{l}\text { Edad- } \\
\text { género }\end{array}$ & Síntoma & $\begin{array}{l}\text { Longitud } \\
\text { de la } \\
\text { necrosis }\end{array}$ & $\begin{array}{c}\text { Hallazgos } \\
\text { endoscópicos }\end{array}$ & $\begin{array}{l}\text { Hallazgos } \\
\text { histológicos }\end{array}$ & Desenlace & $\begin{array}{l}\text { Tratamiento } \\
\text { quirúrgico }\end{array}$ & Enfermedades de base* \\
\hline \multirow[t]{3}{*}{$\begin{array}{l}\text { 62. Shafa S } \\
\text { et al }\end{array}$} & $25 / \mathrm{V}$ & $\begin{array}{l}\text { Melena, anemia aguda } \\
\text { y disfagia }\end{array}$ & $3 / 3$ & $\begin{array}{l}\text { Necrosis total de } \\
\text { esófago, estenosis } 1 / 3 \\
\text { medio de } 5 \mathrm{~cm} \text { largo, no } \\
\text { franqueable }\end{array}$ & & $\begin{array}{l}\text { Estenosis residual a } \\
\text { pesar de las dilataciones }\end{array}$ & & $\begin{array}{l}\text { Cetoacidosis diabética, HTA, } \\
\text { alcohol, cocaína y marihuana }\end{array}$ \\
\hline & $64 / \mathrm{V}$ & $\begin{array}{l}\text { Hematemesis y anemia } \\
\text { aguda, transfusión }\end{array}$ & $3 / 3$ & $\begin{array}{l}\text { Necrosis esofágica total, } \\
\text { circunferencial distal }\end{array}$ & & $\begin{array}{l}\text { Choque, } \mathrm{FMO} \text { y fallece } \\
\text { rápidamente }\end{array}$ & & $\begin{array}{l}\text { HTA, secuelas ECV, } \\
\text { colelitiasis y abuso de alcohol }\end{array}$ \\
\hline & $49 / \mathrm{N}$ & $\begin{array}{l}\text { Melena, FMO, anemia } \\
\text { aguda }\end{array}$ & $1 / 3$ & Necrosis de tercio distal & & $\begin{array}{l}\text { Fallece por infección y } \\
\text { FMO }\end{array}$ & & $\begin{array}{l}\text { Asma, HTA, fiebre, debilidad } \\
\text { y síncope }\end{array}$ \\
\hline $\begin{array}{l}63 . \\
\text { Shimamura Y } \\
\text { et al }\end{array}$ & $38 / \mathrm{N}$ & Hematemesis & $1 / 3$ & $\begin{array}{l}\text { Columnas negras, con } \\
\text { desaparición lecho } \\
\text { vascular superficial }\end{array}$ & $\begin{array}{l}\text { Infiltración por } \\
\text { leucocitos con } \\
\text { hemosiderosis }\end{array}$ & Curación & & Cetoacidosis diabética \\
\hline \multirow[t]{4}{*}{$\begin{array}{l}\text { 64. Singh D } \\
\text { et al }\end{array}$} & $49 / M$ & \multirow{4}{*}{$\begin{array}{l}\text { Náuseas } 3 / 5 \text {, alteración } \\
\text { conciencia } 1 / 5 \text {, } \\
\text { neumomediastino } 1 / 5 \text {, } \\
\text { IRA } 1 / 5 \text {, hematemesis } \\
1 / 5 \text {, ERGE } 1 / 5 \text {, } \\
\text { epigastralgia } 1 / 5 \text {, } \\
\text { síncope } 1 / 5 \text {, hipotensión } \\
1 / 5 \text {, vómito } 2 / 5 \text {, } \\
\text { sangrado digestivo } 2 / 5\end{array}$} & & \multirow[t]{4}{*}{ Esófago negro } & & \multirow[t]{4}{*}{$2 / 5$ fallecen } & & \multirow[t]{4}{*}{$\begin{array}{l}\text { Todos con comorbilidad, } \\
\text { especialmente enfermedad } \\
\text { coronaria, DM, insuficiencia } \\
\text { renal }\end{array}$} \\
\hline & $50 / M$ & & & & & & & \\
\hline & $60 / F$ & & & & & & & \\
\hline & $36 / M$ & & & & & & & \\
\hline $\begin{array}{l}65 . \text { Talebi- } \\
\text { Bakhshayesh } \\
\text { M et al }\end{array}$ & $34 / \mathrm{V}$ & $\begin{array}{l}\text { Hematemesis, dolor } \\
\text { abdominal, náuseas, } \\
\text { vómitos }\end{array}$ & $2 / 3$ & Esófago negro & $\begin{array}{l}\text { Infiltración por } \\
\text { linfocitos, neutrófilos y } \\
\text { eosinófilos, necrosis }\end{array}$ & Curación & & Cetoacidosis diabética \\
\hline $\begin{array}{l}\text { 66. Tanaka K } \\
\text { et al }\end{array}$ & $67 / \mathrm{N}$ & $\begin{array}{l}\text { Vómitos, dolor torácico } \\
\text {--> día } 37 \text { recaída al } \\
\text { suspender IBP }\end{array}$ & $2 / 3$ & & $\begin{array}{l}\text { Exudado difuso y } \\
\text { restos necróticos }\end{array}$ & & & DM, HTA, angina de pecho \\
\hline $\begin{array}{l}\text { 67. Tsao C } \\
\text { et al }\end{array}$ & $73 / \mathrm{V}$ & $\begin{array}{l}\text { Melanemesis al día } \\
\text { siguiente de egreso por } \\
\text { diarrea por antibióticos }\end{array}$ & & & & $\begin{array}{l}\text { Fallece al ingreso, } \\
\text { autopsia: caquéctico, } \\
\text { necrosis aguda } \\
\text { esofágica, colitis } \\
\text { isquémica y } \\
\text { pseudomembranosa }\end{array}$ & & $\begin{array}{l}\text { Dolor abdominal, diarrea } \\
\text { sanguinolenta, pérdida de } 10 \\
\mathrm{~kg}, \mathrm{HTA}, \mathrm{EPOC} \text {, hipovolemia } \\
\text { e infección urinaria }\end{array}$ \\
\hline
\end{tabular}


Tabla 2. Casos descritos en la literatura médica de esofagitis necrotizante (esófago negro). Continuación

\begin{tabular}{|c|c|c|c|c|c|c|c|c|}
\hline $\begin{array}{l}\text { Referencia y } \\
\text { primer autor }\end{array}$ & $\begin{array}{l}\text { Edad- } \\
\text { género }\end{array}$ & Síntoma & $\begin{array}{l}\text { Longitud } \\
\text { de la } \\
\text { necrosis }\end{array}$ & $\begin{array}{c}\text { Hallazgos } \\
\text { endoscópicos }\end{array}$ & $\begin{array}{l}\text { Hallazgos } \\
\text { histológicos }\end{array}$ & Desenlace & $\begin{array}{l}\text { Tratamiento } \\
\text { quirúrgico }\end{array}$ & Enfermedades de base* \\
\hline 68. Tse A et al & $58 / \mathrm{V}$ & $\begin{array}{l}\text { Choque hemorrágico } \\
\text { grave }\end{array}$ & $2 / 3$ & & $\begin{array}{l}\text { Inflamación y necrosis } \\
\text { aguda, transmural en } \\
\text { parches, trombosis } \\
\text { inflamatoria y } \\
\text { vasculitis aguda }\end{array}$ & Curación & $\begin{array}{l}\text { Necrosis } 10 \mathrm{~cm} \text {, } \\
\text { mediastinitis, } \\
\text { derrame pleural } \\
\text { bilateral, } \\
\text { neumomediastino, } \\
\text { perforación distal } \\
\text { esófago }\end{array}$ & $\begin{array}{l}\text { Abuso de alcohol, epilepsia, } \\
\text { ERGE refractaria con } \\
\text { estenosis unión cardial, con } \\
\text { dos stents biodegradable en } \\
20 \text { meses }\end{array}$ \\
\hline $\begin{array}{l}\text { 69. Unuma K } \\
\text { et al }\end{array}$ & $75 / \mathrm{V}$ & $\begin{array}{l}\text { Hallado muerto con } \\
\text { melanemesis }\end{array}$ & $3 / 3$ & & $\begin{array}{l}\text { Necrosis mucosa } \\
\text { extensa }\end{array}$ & $\begin{array}{l}\text { Autopsia: anemia, } \\
\text { esófago negro }\end{array}$ & & Abuso de alcohol \\
\hline $\begin{array}{l}\text { 70. Usmani A } \\
\text { et al }\end{array}$ & & & & & & & & Cetoacidosis diabética \\
\hline $\begin{array}{l}\text { 71. Venara A } \\
\text { et al }\end{array}$ & $75 / \mathrm{V}$ & Muerte súbita & & & Necrosis esofágica & Autopsia & & \\
\hline $\begin{array}{l}\text { 72. Wallberg } \\
\text { ME et al }\end{array}$ & $75 / \mathrm{V}$ & $\begin{array}{l}\text { Vómitos, fiebre, choque, } \\
\text { aspirado gástrico } \\
\text { cuncho de café }\end{array}$ & $3 / 3$ & $\begin{array}{l}\text { Esófago negro, zonas } \\
\text { de esfacelo, erosiones } \\
\text { antrales, restos } \\
\text { hemáticos }\end{array}$ & Necrosis esofágica & Curación & & $\begin{array}{l}\text { HTA, infección urinaria, } \\
\text { choque séptico por } E \text {. coli }\end{array}$ \\
\hline $\begin{array}{l}73 . \\
\text { Watermeyer } \\
\text { GA et al }\end{array}$ & $63 / M$ & & $3 / 3$ & $\begin{array}{l}\text { Esófago negro difuso, } \\
\text { friable, exudado amarillo }\end{array}$ & & Fallece por falla hepática & & Hepatitis tóxica por anti-TBC \\
\hline \multirow[t]{3}{*}{$\begin{array}{l}\text { 74. Worrell } \\
\text { SG et al }\end{array}$} & $84 / M$ & Melena y hematemesis & $2 / 3$ & $\begin{array}{l}\text { Esófago negro entre los } \\
24 \text { y } 36 \mathrm{~cm}\end{array}$ & & Curación & & Cirrosis \\
\hline & $84 / \mathrm{N}$ & $\begin{array}{l}\text { Náuseas, dolor torácico, } \\
\text { y hematemesis }\end{array}$ & $1 / 3$ & Esófago negro & & $\begin{array}{l}\text { Curación con estenosis, } \\
7 \text { dilataciones }\end{array}$ & & DM, HTA \\
\hline & $77 / \mathrm{N}$ & Hematemesis y diarrea & $3 / 3$ & $\begin{array}{l}\text { Esófago negro con } \\
\text { perforación distal }\end{array}$ & & $\begin{array}{l}\text { Fallece al día } 17 \text { por } \\
\text { isquemia intestino } \\
\text { delgado y FMO }\end{array}$ & $\begin{array}{l}\text { TAC: } \\
\text { neumomediastino, } \\
\text { perforación } \\
\text { esofagodistal, } \\
\text { derrame pleural } \\
\text { derecho, } \\
\text { esofaguectomía. } \\
\text { Cervicostomía }\end{array}$ & Cirrosis y DM \\
\hline
\end{tabular}


Tabla 2. Casos descritos en la literatura médica de esofagitis necrotizante (esófago negro). Continuación

\begin{tabular}{|c|c|c|c|c|c|c|c|c|}
\hline $\begin{array}{l}\text { Referencia y } \\
\text { primer autor }\end{array}$ & $\begin{array}{l}\text { Edad- } \\
\text { género }\end{array}$ & Síntoma & $\begin{array}{l}\text { Longitud } \\
\text { de la } \\
\text { necrosis }\end{array}$ & $\begin{array}{c}\text { Hallazgos } \\
\text { endoscópicos }\end{array}$ & $\begin{array}{l}\text { Hallazgos } \\
\text { histológicos }\end{array}$ & Desenlace & $\begin{array}{l}\text { Tratamiento } \\
\text { quirúrgico }\end{array}$ & Enfermedades de base* \\
\hline \multirow[t]{2}{*}{$\begin{array}{l}\text { 75. Wu MH, } \\
\text { Wu HY }\end{array}$} & $67 / \mathrm{V}$ & Melenas & $2 / 3$ & $\begin{array}{l}\text { Esófago negro, úlcera } \\
\text { gástrica y úlcera } \\
\text { duodenal }\end{array}$ & & $\begin{array}{l}\text { Tubo Sengstaken, } \\
\text { perforación esófago a } 24 \\
\text { h y fallece }\end{array}$ & & $\begin{array}{l}\text { Fractura cuello femoral, } \\
\text { hemodiálisis por } \\
\text { hiperpotasemia }\end{array}$ \\
\hline & $46 / \mathrm{V}$ & $\begin{array}{l}\text { Dolor epigástrico, } \\
\text { náuseas y vómito por } \\
7 \text { días, hematemesis y } \\
\text { melenas por } 3 \text { días }\end{array}$ & $\begin{array}{l}1 / 3 \text { distal, } \\
\text { progresó } \\
\text { a todo } \\
\text { esófago }\end{array}$ & Esófago negro & $\begin{array}{l}\text { Necrosis transmural } \\
\text { difusa con exfoliación } \\
1 / 3 \text { distal }\end{array}$ & $\begin{array}{l}\text { 6. día exfoliación } \\
\text { todo esófago, } \\
\text { derrames pleurales, } \\
\text { esofaguectomía, fistula } \\
\text { esofagogastrostomía, } \\
\text { resolución }\end{array}$ & & $\begin{array}{l}\text { Úlcera gástrica con } \\
\text { sangrados frecuentes últimos } \\
2 \text { años, hemorragia úlcera } \\
\text { duodenal }\end{array}$ \\
\hline $\begin{array}{l}\text { 5. Yasuda H } \\
\text { et al }\end{array}$ & $\begin{array}{l}\text { Media } \\
62,5 \text { años }\end{array}$ & & $\begin{array}{l}16 \\
\text { pacientes } \\
1 / 3\end{array}$ & $\begin{array}{l}\text { Necrosis distal y } \mathrm{HH} \\
\text { hallazgos asociados } \\
\text { más frecuentes }\end{array}$ & & & & $\begin{array}{l}\text { Todos con comorbilidad } \\
8 / 16 \text { con AINE, 4/16 DM } \\
\text { cetoacidosis }\end{array}$ \\
\hline $\begin{array}{l}\text { 76. Zacharia } \\
\text { GS et al }\end{array}$ & $62 / M$ & $\begin{array}{l}\text { Náuseas, hematemesis } \\
\text { y dolor retroesternal }\end{array}$ & $1 / 3$ & Esófago negro distal & $\begin{array}{l}\text { Infiltrado inflamatorio } \\
\text { subepitelial con } \\
\text { hemorragia intensa y } \\
\text { tejido necrótico }\end{array}$ & Curación & & DM insulinodependiente, HTA \\
\hline $\begin{array}{l}\text { 77. Živković } \\
\text { V, Nikolić S }\end{array}$ & & & & & & $\begin{array}{l}\text { Fallece por hipotermia, } \\
\text { autopsia }\end{array}$ & & Hipotermia \\
\hline
\end{tabular}

AAA: aneurisma de aorta abdominal; AINE: antiinflamatorios no esteroideos; DHT: deshidratación; DM: diabetes mellitus; DM-I: diabetes mellitus tipo I; DM-II: diabetes mellitus tipo II; DNT: desnutrición; ECV: evento cerebrovascular; EPOC: enfermedad pulmonar obstructiva crónica; ERGE: enfermedad por reflujo gastroesofágico; FMO: falla multiorgánica; GMN: glomerulonefritis; HH: hernia hiatal; HTA: hipertensión arterial; HVDA: hemorragia de vías digestivas altas; ICC: insuficiencia cardíaca congestiva; IRA: insuficiencia renal aguda; IRC: insuficiencia renal crónica; MM II: miembros inferiores; SDRA: síndrome de dificultad respiratoria aguda; SNG: sonda nasogástrica; TAC: tomografía axial computarizada; TBC: tuberculosis. 
sangrado como consecuencia de una hernia hiatal gigante y de ulceraciones de Cameron.

Los hallazgos endoscópicos son diversos y requieren que se descarten otras etiologías que producen alteraciones similares, como la ingestión de cáusticos, otros tóxicos o carbón, infecciones, radioterapia o melanoma metastásico, entre otros $(3,10)$. Los hallazgos pueden ir desde membranas blanquecinas, que al removerlas dejan una mucosa grisácea o negruzca, hasta necrosis difusa con exudados amarillentos adherentes, ulceración en la mayoría y aspecto negruzco o grisáceo, demarcado por la unión gastroesofágica, con región cardial de aspecto usual, como se describen en nuestros casos. El espectro de lesiones asociadas es amplio, como hemorragia gástrica o duodenal, ulceraciones y perforaciones, representadas en la casuística general con úlceras duodenales $(10 ; 4 \%)$, gástricas $(2 ; 1 \%)$, necrosis isquémica duodenal $(2 ; 1 \%)$; sangrado activo $(4 ; 2 \%)$, perforación esofágica $(3 ; 1,3 \%)$ y duodenal $(1 ; 0,5 \%)$ (Tabla 1).

El desenlace fue muy variable, aunque favorable en el segundo de nuestros pacientes, con normalización de la mucosa esofágica en el control un mes después. El tercer paciente permaneció asintomático diez semanas después y rechazó la realización de controles; no obstante, desconocemos la evolución que presentaron tres de los pacientes. Sin embargo, en la serie general, la mortalidad alcanzó el $32 \%$, la curación ocurrió en el $38 \%$ y las complicaciones con resolución parcial en el resto (Tabla 1), que incluyeron, entre otras, estenosis residual, que resolvió en dos tercios de los pacientes con las dilataciones y que en el $4 \%$ requirió algún tratamiento quirúrgico.

La comorbilidad asociada es el hallazgo común en la mayoría de los casos de la literatura, enmarcada en pacientes de alto riesgo, riesgo que está determinado, a su vez, por la edad de presentación, las enfermedades debilitantes agudas y crónicas, el compromiso hemodinámico y la disfunción multiorgánica (Tabla 1), igualmente presentes en nuestros pacientes, con hiponatremia, hipocloremia e insuficiencia renal aguda prerrenal (caso 1); hernia hiatal gigante de $9 \mathrm{~cm}$ y enfermedad por reflujo gastroesofágico grave, con antecedentes de sangrado en dos ocasiones (caso 2); neumonía basal en evolución, trombocitopenia severa, insuficiencia renal crónica agudizada y coledocolitiasis con obstrucción de la vía biliar (caso 3); y trombosis mesentérica (caso 4). En la Tabla 1 se aprecia la comorbilidad en la serie global, en la que se describe generalmente como enfermedades graves agudas en el $78 \%$ de 157 pacientes, y en los 104 restantes, en quienes se detallan con hemorragia y choque, hipertensión arterial, cardiopatía, insuficiencia hepática, cetoacidosis diabética, insuficiencia renal, septicemia y alcoholismo, entre otros, con más de una patología en la mayoría de los pacientes.

\section{Histopatología y etiología}

En nuestro paciente MDA (caso 2), las biopsias del esófago distal muestran esofagitis aguda, con extensa necrosis mucosa y submucosa con capa muscular viable y cambios epiteliales reactivos. Los hallazgos histológicos se obtienen en unos pocos casos (15\%), ya sea en biopsias tomadas durante la endoscopia diagnóstica, a partir de la pieza quirúrgica o de autopsia $(3,10)$, obtenidas en el $15 \%$ de la serie global, con demostración de la participación de la submucosa y muscular propia, compromiso profundo de la pared esofágica y presencia de necrosis en casi todos los casos, inflamación, isquemia, no solamente de esófago, sino también de estómago y duodeno, y trombosis (Tabla 1).

La gravedad y la profundidad de la lesión fueron demostradas por la evolución clínica y las complicaciones asociadas, como la perforación esofágica, la infección mediastinal, el absceso, la fístula y la estenosis esofágica, que se resuelven solamente en el $40 \%$ de los casos, a pesar de las dilataciones (Tabla 1) y, por supuesto, la elevada mortalidad del $32 \%$.

Acerca de la etiología, en cuanto a los casos reportados y a la literatura revisada, se cree que está asociada con hipoperfusión a nivel de la mucosa esofágica, ocasionada por disminución del flujo sanguíneo por las diferentes ramas arteriales. Esto da origen a una isquemia que, posteriormente, lleva a necrosis. La disminución del flujo sanguíneo está asociada con la presencia de enfermedades de base, las cuales pueden transcurrir con alteración en la sensibilidad y la microvasculatura, desequilibrio hemodinámico y electrolítico, hipersensibilidad a medicamentos, como los antibióticos y los AINE, alcoholismo y reflujo ácido $(3,7,14)$, como puede corroborarse en la comorbilidad asociada y en el compromiso multiorgánico y hemodinámico frecuente, agravado por el sangrado digestivo que lleva al paciente a consultar (Tabla 1). Adicionalmente, los hallazgos de isquemia y trombosis en los estudios histopatológicos están a favor de esta hipótesis. No existen estudios experimentales que comprueben estas hipótesis.

\section{Pronóstico}

El pronóstico depende de la gravedad del compromiso del estado general y de la edad avanzada. Un grupo de pacientes que fallecieron lo hicieron en las primeras seis horas (cuatro casos) y a dos días del ingreso (dos casos), además de los que presentaron muerte súbita o fueron hallados inconscientes (seis casos). La extensión de la necrosis parece tener relación con el desenlace fatal, dado que solo dos pacientes con compromiso de un tercio o de la mitad del esófago fallecieron, mientras que el compromiso fue de 
dos tercios de la totalidad del esófago en los 29 restantes, en los que su extensión fue descrita. La enfermedad de base fue la responsable de la mortalidad en un gran número de los pacientes y los autores la describieron como la causa principal en 22 pacientes.

Como factores de riesgo se pueden mencionar la edad avanzada, el género masculino, la extensión de la necrosis y la comorbilidad asociada: hemorragia, choque y sepsis, hipertensión arterial, cardiopatía, insuficiencia hepática y renal, cetoacidosis diabética y alcoholismo.

\section{Tratamiento}

Los autores son enfáticos y unánimes en afirmar que no existe un tratamiento específico de la esofagitis necrotizante, ante la ausencia de un factor etiológico definido y su asociación con múltiples entidades que pueden conducir a dicha situación y potenciarse entre sí para inducir la lesión esofágica con isquemia, trombosis y necrosis de la mucosa y de la pared esofágica. De esta manera, se recomiendan las medidas generales de soporte para el manejo de las enfermedades de base desencadenantes de la hipoperfusión (choque, sepsis, insuficiencia cardíaca, renal y hepática y falla multiorgánica) y restablecer el equilibrio hemodinámico, ácido-básico y metabólico; en caso de anemia, transfundir glóbulos rojos empaquetados; también administrar inhibidor de la bomba de protones intravenoso para el control de la lesión ácidopéptica asociada en algunos o por el agravamiento de la lesión por su presencia $(3,7-11,14)$, e incluso el uso de stents para el manejo del sangrado activo profuso, utilizado en un caso como método de rescate (54). Naturalmente, el manejo de las complicaciones médicas y quirúrgicas pertinentes, como la sobreinfección, las perforaciones y las fístulas; la realización de dilataciones en las estenosis y la colocación de stents en caso de pobre respuesta $(34,35,72)$, hasta esofaguectomía $(35,45,78,79)$ en pacientes con complicaciones graves (Tabla 1) (3, 6-11, 14).

\section{CONCLUSIONES}

La esofagitis necrotizante es un trastorno esofágico grave, raro, con una elevada tasa de mortalidad y de complicaciones. La elevada comorbilidad que le acompaña es, al parecer, la desencadenante y causante de la mortalidad en un grupo importante, que junto con la edad avanzada, el género masculino y la extensión son factores de riesgo y pronóstico. El enfoque terapéutico debe ser, por tanto, interdisciplinario y estar dirigido al restablecimiento del equilibrio hemodinámico, ácido-básico, metabólico y ácido-péptico, y al manejo de las complicaciones infecciosas. Las complicaciones graves son frecuentes y requieren de evaluación cercana y manejo oportuno por el riesgo vital.

\section{Financiación}

Se declara que no existieron apoyos financieros para la ejecución y el análisis de este estudio.

\section{Conflictos de interés}

Este estudio no ha sido presentado previamente en ninguna reunión o enviado a publicación alguna.

\section{REFERENCIAS}

1. Brennan JL. Case of extensive necrosis of the oesophageal mucosa following hypothermia. J Clin Pathol. 1967;20:5814. doi: 10.1136/jcp.20.4.581.

2. Lee KR, Shark E, Shaw FE. Esophageal infarction complicating spontaneous rupture of the thoracic aorta. JAMA. 1977;237:1233-4. doi: 10.1001/jama.237.12.1233.

3. Day A, Sayegh M. Acute oesophageal necrosis: a case report and review of the literature. Int J Surg. 2010;8(1):6-14. doi: 10.1016/j.ijsu.2009.09.014.

4. Postlethwait RW, Musser AW. Changes in the esophagus in 1,000 autopsy specimens. J Thorac Cardiovasc Surg. 1974;68:953-6 (Abstract).

5. Etienne JP, Roge J, Delavierre P, Veyssier P. Esophageal necrosis of vascular origin. Sem Hop. 1969;45:1599-606 (Abstract).

6. Jacobsen NO, Christiansen J, Kruse A. Incidence of oesophageal necrosis in an autopsy material. Brief report. APMIS. 2003;111(5):591-4. doi: 10.1034/j.16000463.2003.1110509.x.

7. Gurvits GE. Black esophagus: Acute esophageal necrosis syndrome. World J Gastroenterol. 2010;26:3219-25. doi: 10.3748/wjg.v16.i26.3219.

8. Grudell ABM, Mueller PS, Viggiano TR. Black esophagus: report of six cases and review of the literature, 1963-2003. Dis Esophagus. 2006;19:105-10. doi: 10.1111/j.14422050.2006.00549.x.

9. Ramos R, Mascarenhas J, Duarte P, Vicente C, Casteleiro C. Esofagitis necrosante aguda: análisis retrospectivo. Rev Esp Enferm Dig (Madrid). 2008;100(9):583-5. doi: 10.4321/ S1130-01082008000900010.

10. Gómez LJ, Barrio J, Atienza R, Fernández-Orcajo P, Mata L, Saracíbar E, et al. Esofagitis necrotizante aguda. Una patología poco conocida. Rev Esp Enferm Dig (Madrid). 2008;100(11):701-5.

11. Yasuda H, Yamada M, Endo Y, Inoue K, Yoshiba M. Acute necrotizing esophagitis: Role of nonsteroidal anti-inflammatory drugs. J Gastroenterol. 2006;41:193. (Abstract). doi: 10.1007/s00535-005-1741-6.

12. Ángel LA, Boada D, Villalba MP. Evolución epidemiológica de la enfermedad ácido péptica en un Centro de endoscopia diagnóstica de Bogotá entre 1993 y 2007. Rev Col Gastroenterol. 2010;25(2):139-53. 
13. Augusto F, Fernandes V, Cremers MI, Oliveira AP, Lobato $\mathrm{C}$, Alves AL, et al. Acute necrotizing esophagitis: A large retrospective case series. Endoscopy. 2004;36:411-5. doi: 10.1055/s-2004-814318.

14. Gurvits GE, Shapsis A, Lau N, Gualtieri N, Robilotti JG. Acute esophageal necrosis: a rare syndrome.J Gastroenterol. 2007;42:29-38. doi: 10.1007/s00535-006-1974-z.

15. Abed J, Mankal P, Judeh H, Kim S. Acute Esophageal Necrosis: A Case of Black Esophagus Associated with Bismuth Subsalicylate Ingestion. ACG Case Rep J. 2014;1(3):131-3. doi: 10.14309/crj.2014.27.

16. Abu-Zaid A, Solimanie S, Abudan Z, Al-Hussaini H, Azzam A, Amin T. Acute esophageal necrosis (black esophagus) in a 40-year-old man. Ann Saudi Med. 2015;35(1):80-1. doi: 10.5144/0256-4947.2015.80.

17. Akkinepally S, Poreddy V, Moreno A. Black esophagus. Cleve Clin J Med. 2009;76(7):400. doi: 10.3949/ ccjm.76a.08102.

18. Altenburger DL, Wagner AS, Li S, Garavaglia J. A Case of Black Esophagus With Histopathologic Description and Characterization. Arch Pathol Lab Med. 2011;135:797-8.

19. Ambrosio MR, Rocca BJ, Mastrogiulio MG, Lutfi A, Lazzi S, Tripodi SA. A case of acute esophageal necrosis (black esophagus) in a young man with Down syndrome. Esophagus. 2014;11(2):136. (Abstract). doi: 10.1007/ s10388-013-0396-2.

20. Barnes T, Yan S, Kaakeh Y. Necrotizing Esophagitis and Bleeding Associated With Cefazolin. Pharmacother. 2014;48(9):1214-8. (Abstract). doi: 10.1177/ 10600280 14537038.

21. Bremholm L, Winkel R, Born P, Suku ML. Acute esophageal necrosis. Ugeskr Laeger. 2009; 171(45):3282-3. (Abstract).

22. Cameron PA, Schweiger F. Forsaken Foregut: Case Report of Simultaneous Black Esophagus and Ischemic Cholangiopathy. Case Reports in Medicine, Published 24 April 2017. doi: 10.1155/2017/8362613.

23. Caravaca-Fontán F, Jimenez S, Fernández-Rodríguez A, Marcén R, Quereda C. Black esophagus in the early kidney post-transplant period. Clin Kidney J. 2014;7:613-4. doi: $10.1093 / \mathrm{ckj} / \mathrm{sfu} 094$.

24. Casta-o-Llano R, Matar-Khalil O, Cárdenas-Vásquez A, Ruiz M, Juliao F, Sanín E et al. El esófago negro: ¿Patología infrecuente o desconocida? Rev Col Gastroenterol. 2006;21(1):62-7.

25. Choi EJ, Lee $\mathrm{SH}$, Lee SH. A case of acute necrotizing esophagitis. Korean J Gastroenterol. 2010;56(6):373-6. (Abstract). doi: 10.4166/kjg.2010.56.6.373.

26. De Palma GD, Persico M, Forestieri P. A case of coffeeground emesis in an elderly patient. Clin Case Rep. 2014;2(1):18. doi: 10.1002/ccr3.45.

27. Edling $P$, Thomsen $H$. Acute oesophageal necrosis in patients with diabetic ketoacidosis. Ugeskr Laeger. 2013;175(48A) (Abstract).

28. Eren B, Turkmen N, Fedakar R. Black esophagus: a rare autopsy case. Bratisl Lek Listy. 2010;111(7):414-5 (Abstract).
29. Fernández R, Álvarez L, Puertas F, González A, García V, López V. Black esophagus (acute esophageal necrosis) after spinal anesthesia. Rev Esp Anestesiol Reanim. 2014;61(7):401-3. doi: 10.1016/j.redar.2013.06.001.

30. Fernández-Carrillo C, Barrios-Peinado C, Pons-Renedo F. Acute necrotizing esophagitis in a patient with femur fracture. Rev Esp Enferm Dig (Madrid). 2011;103(8):436-7. doi: $10.4321 /$ S1130-01082011000800012.

31. Galanopoulos M, Anastasiadis S, Archavlis E, Mantzaris GJ. Black esophagus: an uncommon cause of upper gastrointestinal bleeding. Intern Emerg Med. 2015;11(7):1019-20. doi: 10.1007/s11739-015-1360-z.

32. Galtés I, Gallego MA, Esgueva R, Martin-Fumadó C. Necrosis esofágica aguda (esófago negro). Rev Esp Enferm Dig (Madrid). 2016;108(3):154-5.

33. Garas G, Wou C, Sawyer J, Amygdalos I, Gould S. Acute oesophageal necrosis syndrome. BMJ Case Reports. 2011. doi:10.1136/bcr.10.2010.3423.

34. Gómez ÁA, Guerrero D, Hani AC, Caadas R. Acute necrotizing esophagitis (black esophagus) with secondary severe stenosis]. Rev Gastroenterol Peru. 2015;35(4):349-54 (Abstract).

35. Gómez V, Propst JA, Francis DL, Canabal JM, Franco PM. Black esophagus: an unexpected complication in an orthotopic liver transplant patient with hemorrhagic shock. Dig Dis Sci. 2014;59(10):2597-9. doi: 10.1007/s10620014-3176-5.

36. Groenveld RL, Bijlsma A, Steenvoorde P, Ozdemir A. A black perforated esophagus treated with surgery: Report of a case. World J Gastrointest Surg. 2013;5(6):199-201. doi: 10.4240/wjgs.v5.i6.199.

37. Gurvits GE, Cherian K, Shami MN, Korabathina R, El-Nader EM, Rayapudi K, et al. Black esophagus: new insights and multicenter international experience in 2014. Dig Dis Sci. 2015;60(2):444-53 (Abstract). doi: 10.1007/ s10620-014-3382-1.

38. Hejna P, Ublová M, Voříšek V. Black esophagus: acute esophageal necrosis in fatal haloperidol intoxication. J Forensic Sci. 2013;58(5):1367-9 (Abstract). doi: 10.1111/1556-4029.12151.

39. Hermet A, Lidove O, Grimbert S, Zeller V, Ducroquet F, Ziza JM. Black esophagus. Rev Med Int. 2014;35(6):393-5 (Abstract). doi: 10.1016/j.revmed.2013.06.006.

40. Hong JW, Kim SU, Park HN, Seo JH, Lee YC, Kim H. Black esophagus associated with alcohol abuse. Gut Liver. 2008;2(2):133-5. doi: 10.5009/gnl.2008.2.2.133.

41. Iorio N, Bernstein GR, Malik Z, Schey R. Acute Esophageal Necrosis Presenting With HenochSchönlein Purpura. ACG Case Rep J. 2015;3(1):17-9. doi: 10.14309/crj.2015.87.

42. Ishibashi Y, Matsuzono E, Goda T, Yokoyama F, Sugai N, Seki H, et al. Black esophagus due to acute necrotizing esophagitis: four case reports. Nihon Shokakibyo Gakkai Zasshi. 2011;108(5):759-8 (Abstract).

43. Kanaparthi C, Singhal S, Guillaume C, Sharma S, Anand S. Acute gastrointestinal necrosis: "black esophagus" or "black gut”. Trop Gastroenterol. 2011;32(4):323-6. 
44. Karaahmet F, Hamamci M, Coban S, Yuksel I. A rare entity with coffee-ground vomit in elderly adults: black esophagus. J Am Geriatr Soc. 2014;62(8):1610-1. doi: 10.1111/ jgs. 12952 .

45. Kim IK, Kim JS, SongIS.A Case of Acute Esophageal Necrosis with Gastric Outlet Obstruction. Korean J Gastroenterol. 2010;56:314-8. doi: 10.4166/kjg.2010.56.5.314.

46. Kim YH, Choi SY. Black esophagus with concomitant candidiasis developed after diabetic ketoacidosis. World J Gastroenterol. 2007;13(42):5662-3. doi: 10.3748/wjg.v13.i42.5662.

47. Köksal AS, Eminler AT, Parlak E, Uslan MI, Cücen E. Black esophagus and duodenal perforation: more than an incidental association. Acta Gastroenterol Belg. 2015;78(2):253-4 (Abstract).

48. Kwon HJ, Park SH, Ahn JH, Lee TH, Lee CK. Acute esophageal necrosis occurring in a patient undergoing percutaneous coronary intervention. Korean J Intern Med. 2014;29:379-82. doi: 10.3904/kjim.2014.29.3.379.

49. Lahbabi M, Ibrahimi A, Aqodad N. Acute esophageal necrosis: a case report and review. Pan Afr Med J. 2013;14:109. doi:10.11604/pamj.2013.14.109.2000.

50. Lin MC, Chu HC, Chen PJ. Clinical picture: Black oesophagus. QJM. 2015;108(10):839-40. doi: 10.1093/qjmed/hcv075.

51. Maher MM, Nassar MI. Black esophagus: a case report. Cases J. 2008;1:367. doi: 10.1186/1757-1626-1-367.

52. Maroy B. Black esophagus complicating variceal bleeding. Endoscopy. 2013;45(Suppl 2):E237. doi: 10.1055/s-00301256630.

53. McLaughlin CW, Person TD, Denlinger CE, Charleston SC. Management of acute esophageal necrosis syndrome. J Thorac Cardiovasc Surg. 2011;141:e23-e24. doi: 10.1016/j. jtcvs.2010.12.001.

54. Messner Z, Gschwantler M. Use of the Ella Danis stent in severe esophageal bleeding caused by acute necrotizing esophagitis. Endoscopy 2014;46:E225-6. doi: 10.1055/s0034-1365384.

55. Nikolić S, Zivković V. Black esophagus--case report. Srp Arh Celok Lek. 2014;142(1-2):94-8 (Abstract). doi: 10.2298/ SARH1402094N.

56. Pastuszak M, Groszewski K. Endoscopic image of "black esophagus"--case report. Pol Merkur Lekarski. 2009;26(155):468-71 (Abstract).

57. Pereira O, Figueira-Coelho J, Picado B, Neves-Costa J. Black Oesophagus. BMJ Case Rep. 2013. doi: 10.1136/bcr-2012008188

58. Pierini A, Imhof H, Burlando E, Gianinetti L, Pierini L. Acute necrotizing esophagitis. Case report. Acta Gastroenterol Latinoam. 2013;43(2):130-2 (Abstract).

59. Plaza-Santos R, Froilán-Torres C, Castro-Carbajo P, MartínChavarri S, Gea-Rodríguez F, Suárez de Parga JM. Esofagitis aguda necrosante asociada a coma diabético hiperosmolar. J Gastrohep. 2009;32(4):322-3. doi: 10.1016/j.gastrohep.2008.09.026.

60. Pramparo SE, Ruiz PM, Flores-Valencia R, Rodeyro J. Esofagitis necrotizante aguda: una entidad inusual. Medicina (Buenos Aires). 2010;70:524-6.
61. Rigolon R, Fossà I, Rodella L, Targher G. Black esophagus syndrome associated with diabetic ketoacidosis. World J Clin Cases. 2016;4(2):56-9. doi: 10.12998/wjcc.v4.i2.56.

62. Rodrigo M, Gorraiz BE, Merino C, Eguaras J, Elizalde JM, Soler W. Acute necrotizing esophagitis in an unstable patient. An Sist Sanit Navar. 2009;32(1):113-6.

63. Román-Fernández A, López-Álvarez A, Fossati-Puertas S, Areán-González I, Varela-García O, Via-o-López PM. Black esophagus (acute esophageal necrosis) after spinal anesthesia. Rev Esp Anestesiol Reanim. 2014;61(7):401-3. doi: 10.1016/j.redar.2013.06.001.

64. Salem GA, Ahluwalia S, Guild RT. A case of acute oesophageal necrosis (AEN) in a hypothermic patient. The grave prognosis of the black oesophagus. Arab J Gastroenterol. 2015; 16(34):136-8 (Abstract). doi: 10.1016/j.ajg.2015.05.002.

65. Santos VM, Villaça RB, Gouvêa IP, Sá DAR. Comentario a la nota clínica: Esofagitis necrotizante aguda en paciente inestable. An Sist Sanit Navar. 2009;32(3):439-42.

66. Shafa S, Sharma N, Keshishian J, Dellon ES. The Black Esophagus: A Rare But Deadly Disease. ACG Case Rep J. 2016;3(2):88-91. doi: 10.14309/crj.2016.9.

67. Shimamura Y, Nakamura K, Ego M, Omata F. advanced endoscopic imaging of black esophagus. Can J Gastroenterol Hepatol. 2014;28(9):471-2. doi: 10.1155/2014/620709.

68. Singh D, Singh R, Laya AS. Acute esophageal necrosis: a case series of five patients presenting with "Black esophagus”. Indian J Gastroenterol. 2011;30(1):41-5 (Abstract). doi: $10.1007 / \mathrm{s} 12664-011-0082-\mathrm{z}$.

69. Talebi-Bakhshayesh M, Samiee-rad F, Zohrenia H, Zargar A. Acute Esophageal Necrosis: A Case of Black Esophagus with DKA. Arch Iran Med. 2015;18(6):384-5.

70. Tanaka K, Toyoda H, Hamada Y, Aoki M, Kosaka R, Noda $\mathrm{T}$, et al. Relapse of acute necrotizing esophagitis. Endoscopy. 2007;39:E305. doi: 10.1055/s-2007-966789.

71. Tsao C, Thomas L, McGoey RR. Black esophagus detected at autopsy in a patient with abdominal pain and bloody diarrhea. Diagnosis: Acute esophageal necrosis, ischemic and pseudomembranous colitis. J La State Med Soc. 2014;166(4):188-190 (Abstract).

72. Tse A, Basu S, Ali H, Hamouda A. Black necrotic oesophagus following the use of biodegradable stent for benign oesophageal stricture. J Surg Case Rep. 2015;7:1-3. doi: $10.1093 /$ jscr/rjv072.

73. Unuma K, Harada K, Funakoshi T, Uemura K. Sudden death of an alcoholic elderly man with acute esophageal necrosis (black esophagus). Forensic Sci Int. 2011;212(1-3):e15-7 (Abstract). doi: 10.1016/j.forsciint.2011.05.024.

74. Usmani A, Samarany S, Nardino R, Shaib W. Black esophagus in a patient with diabetic ketoacidosis. Conn Med. 2011;75(8):467-8.

75. Venara A, Pavageau AH, Maillart CR, Jousset N. Sudden death due to black esophagus: a case report. Am J Forensic Med Pathol. 2013;34(1):16-7 (Abstract). doi: 10.1097/ PAF.0b013e3182717b98.

76. Wallberg ME, Young P, Finn BC, Thomé M, Chueco AA, Villarejo F. Esófago negro secundario a esofagi- 
tis necrotizante aguda. Reporte de un caso clínico. Rev Med Chile. 2009;137:672-4. doi: 10.4067/S003498872009000500011.

77. Watermeyer GA, Shaw JM, Krige JEJ. Gastrointestinal: Acute necrotizing esophagitis. J Gastroenterol Hepatol 2007;22:1162. doi: 10.1111/j.1440-1746.2007.05013.x.

78. Worrell SG, Oh DS, Greene CL, DeMeester SR, Hagen JA. Acute Esophageal Necrosis: A Case Series and LongTerm Follow-Up. Ann Thorac Surg. 2014;98:341-2. doi: 10.1016/j.athoracsur.2013.09.023.
79. Wu MH, Wu HY. Incremental change in acute esophageal necrosis: report of two cases. Surg Today. 2014;44(2):3635. doi: 10.1007/s00595-013-0526-4.

80. Zacharia GS, Sandesh K, Ramachandran TM. Acute Esophageal Necrosis: An Uncommon Cause of Hematemesis. Oman Med J. 2014;29(4):302-4. doi: 10.5001/omj.2014.79.

81. Živković V, Nikolić S. The unusual appearance of black esophagus in a case of fatal hypothermia: a possible underlying mechanism. Forensic Sci Med Pathol 2013;9(4):613-4 (No abstract). doi: 10.1007/s12024-013-9445-3. 